\title{
La política nuclear y de no-proliferación de Brasil*
}

\author{
Abreviaturas \\ AEC \\ GBTN \\ GENTEX \\ GESP \\ GNE \\ GNEN \\ CNPq \\ CFI \\ CSN \\ CTA \\ DFVIR \\ ELECTROBRAS \\ ESP \\ IAE (CTA) \\ IAEA \\ IEA \\ IEN \\ IHT \\ IME \\ IPEN \\ JB \\ $\mathrm{kg}$ \\ $\mathrm{km}$ \\ KW \\ KWU \\ LEA (C'TA) \\ LARR \\ LAWR \\ MRBM \\ MW \\ NNWS \\ NPT \\ NSG \\ Atomic Energy Commission (US). \\ Companhia Brasileira de Tecnologia Nuclear. \\ Centro Tecnologico do Exército. \\ Companhia Eléctrica de São Paulo. \\ Conselho Nacional de Energía. \\ Comissão Nacional de Energia Nuclear. \\ Conselho Nacional de Pesquisas. \\ Comissão Parlamentar de Inquérito do Senado Federal so- \\ bre o Acordo Nuclear do Brasil com a República Fede- \\ ral de Alemanhia. \\ Conselho de Segurança Nacional. \\ Gentro Técnico Aéroespacial. \\ Deutsche Forschungs-und Versuchsanstalt für Luftund \\ Raumfahrttechnik. \\ Centrais Elétricas Brasileiras. \\ O Estado de São Paulo. \\ Instituto de Atividades Espaciais. \\ International Atomic Energy Agency. \\ Instituto de Energia Atômica. \\ Instituto de Engenharia Nuclear. \\ International Herald Tribune. \\ Instituto Militar de Engenharia. \\ Instituto das Pesquisas Energéticas e Nucleares. \\ Jornal do Brasil. \\ Kilogramo. \\ Kilómetro. \\ Kilowatt. \\ Kraftwerk Union. \\ Laboratorio de Estudios Avançados. \\ Latin American Regional Report. \\ Latin America Weekly Report. \\ Medium-Range Ballistic Missile. \\ Megawatt. \\ Non-Nuclear Weapon State. \\ Tratado de No-Proliferación (Nuclear). \\ Grupo de Proveedores Nucleares.
}

* Me gustaría expresar mis agradecimientos al señor Gunnar Wiegand por su excelente investigación y sus muy valiosas sugerencias para la preparación de este capítulo. 


NUCLAM
NUGLEBRAS
NUCLEI
NUCLEMON
NUCLEN
NUCLEP
NUCON
NUSTEP
NWS
OPANAL (IT)
PETROBRAS
PNE
SBPC
SIPRON
SNI
TT

\author{
Nuclebrás Auxiliar de Mineraçao. \\ Emprera Brasileira de Energia Nuclear. \\ Nuclebrás Enriquecimiento Isotópico. \\ Nuclebrás de Monazita e Associađos. \\ Nuclebrás Engenharia. \\ Nuclebrás Equipamentos Pesados. \\ Nuclebrás Construtora de Centrais Elétricas. \\ Trenndüsen Entwicklungs-und \\ Patentverwertungsgesellschaft. \\ Nuclear Weapon State. \\ Organismo para la Proscripción de las Armas Nucleares \\ en la América Latina. \\ Petroleo Brasileiro. \\ Peaceful Nuclear Explosion. \\ Sociedade Brasileira para o Progresso da Giência. \\ Serviço de Proteção ao Programa Nuclear. \\ Serviço Nacional de Intelligência. \\ Tratado de Tlatelolco.
}

\section{INTRODUCCIón}

\subsection{Actitud hacia la no-proliferación}

Todos los gobiernos brasileños - cualquiera haya sido su orientación politica- se han negado a firmar el Tratado de No-proliferación (NPT). Los esfuerzos hacia la no-proliferación se perciben como un medio por el cual los países desarrollados monopolizan Ia investigación nuclear y de ese modo impiden a los estados menos desarrollados de adquirir una de las tecncologías del futuro más sofisticadas y promisorias.

Sin embargo, los regímenes de no-proliferación regional son más aceptables porque excluyen a las potencias nucleare; establecidas. Brasil fue uno de los inic:adores del Tratado de Tlatelolco de 1967 (TT), que se suponía iba a crear en América Latina una zona libre de armas nucleares. (Ver secciones 2.1 y 5.1). A pesar de esto, la actitud de Brasil hacia el Tratado se ha vuelto ambivalente desde entonces. Sólo ratificó el Tratado después de lograr Ia inclusión de una cláusula que permite "las explosiones nucleares pacíficas" (PNEs). Más aún, el Tr no se ha puesto en práctica en Brasil porque no se ha abolido la disposición (Art. 28, I), que requiere que todos los e:tados latinoamericanos ratifiquen el tratado antes de que entre en vigor, $y$ aún hay algunos que ni siquiera lo han firmado.

Un hecho positivo ha sido la cooperación nuclear con Argentina. que empezó en 1980 y que es descrita en la sección 4.3.

1.2. Preocupación respecto a la proliferación.

Desde 1975, cuando Brasil firmó un acuerdo con Ia República. Fede- 
ral de Alemania (RFA) que suministraba la transferencia de un amplio rango de tecnologías nucleares, ha existido preocupación entre los países desarrollados. En 1985, con el cambio hacia un gobierno democrático, surgieron esperanzas de que Brasil pudiera cambiar su tradicional posición sobre no-proliferación. Se esperaba que un gobierno democrático estuviera menos preocupado del status internacional de su país $y$, por lo tanto, estuviera más dispuesto a someter al país al NPT. La drástica desaceleración del programa de energía nuclear de 1975, y en particular el retraso de la construcción de" la planta de reprocesamiento planeada, parecen apoyar estas esperanzas.

Esta visión optimista, sin embargo, no toma en consideración el amplio "programa paralelo" que se inició a principios de los 80 s y que se lleva a cabo en primitivos centros de investigación nuclear, operando al margen de las medidas de seguridad internacionales. A pesar del gobierno democrático, los militares aún tienen un firme control sobre estos proyectos de investigación, que están destinados, aparentemente, a construir las instalaciones necesarias para el desarrollo de explosivos nucleares. Es difícil conseguir información oficial respecto a estos proyectos, puesto que el gobierno actual está ansioso por mantener al público desinformado, por las mismas razones que el gobierno militar anterior. En la esfera internacional, el gobierno ha tratado de evitar conflictos con esos paises que han proveído partes para las instalaciones usadas en la continuación del "programa paralelo". A nivel regional, el gobierno está obligado a ser discreto debido a su antigua rivalidad con Argentina, que ha sido capaz de mantener su liderazgo tanto en la investigación nuclear como en el uso de la energía nuclear. A nivel nacional, el gobierno ha tratado de evitar debates públicos sobre la utilidad de la colocación de recursos presupuestarios para propósitos nucleares en tiempos de déficit públicos en aumento y de fuertes críticas al pro-s grama de energía nuclear.

El curso de la política nuclear brasileña está cambiando. En lugar de la masiva transferencia de tecnología nuclear desde RFA, que se ha disminuido a un mínimo, Brasil está luchando con mayor esfuerzo por dominar el ciclo nuclear con medios primitivos $y$, al mismo tiempo, por adquirir una amplia gama de tecnologías nucleares.

\subsection{Capacidades nucleares actuales.}

\section{Producción de energia}

Después de firmar el 27 de junio de 1975 su acuerdo con la RFA, Brasil realizó al principio progresos significativos al crear una infraestructura de energía nuclear. Pero las limitaciones financieras, 
Wolf Grabendorff / La polftica nuclear y de no-proliferación de Brasil

los problemas técnicos y la crítica del público desaceleraron su implementación.

Los componentes principales del acuerdo incluían la mineria y exploración de uranio, el enriquecimiento del uranio, la fabricación de combustible, el reprocesamiento del combustible ya usado, y plantas nucleares. Más aún, el personal brasileño había de recibir entrenamiento extensivo de firmas de Alemania Federal, para preparar la eventual toma de todo el programa nuclear por parte de Brasil y su crecimiento como fabricante de componentes nucleares. Se esperaba que la totalidad del paquete iba a llegar a $10 \mathrm{mil} \mathrm{mi-}$ llones de dólares, y que el financiamiento para la primera etapa provendría de un consorcio de bancos de Alemania Federal.

Se han realizado considerables progresos en la localización y clasificación de las reservas de uranio, que se calculaba que alcanzaban a 310.000 toneladas en $1985^{1}$. Las reservas de torio se estimaban en 80.000 toneladas. Sin embargo, se cree que los reactores de torio no tendrán significación comercial hasta bastante entrado el próximo siglo?.

La tecnología brasileña de enriquecimiento de uranio está basada en el proceso de enriquecimiento por pitón a chorro, que aún no es operacional a escala industrial en ninguna parte. Este era el único proceso disponible para transferencia, porque el gobierno brasileño exigía control total sobre el proceso, y esta era la única tecnología de enriquecimiento de uranio sobre la que los alemanes tenían control total. El Reino Unido y los Países Bajos (los socios de la RFA en URENCO, el consorcio de enriquecimiento de uranio) no aceptarían exportar a un país no firmante del NPT las tecnogías más económicas de enriquecimiento por centrifugado.

Según el acuerdo, Brasil había de recibir una planta de enriquecimiento en Resende a un costo de $\$ 282$ millone: ${ }^{3}$. Esta planta hubiera tenido una capacidad de enriquecimiento anual de 300 toneladas de uranio a un 3,2 por ciento y debía ser construida dentro de un plazo de cinco años.

Sin embargo, no será hasta 1986 que la primera serie de plantas empiece a operar, con una capacidad de alrededor de 60 toneladas de uranio por año, enriquecida hasta un 0,9 por ciento. Sólo esta parte ya costará $\$ 300$ millones 4 . Produciendo 300 toneladas al año, la planta consumirá $1.500 \mathrm{Mw}$ de electricidad, equivalentes a toda la producción de una planta de energía mediana ${ }^{5}$. Es por lo tanto

ISouth, septiembre de 1985, p. 21.

"David J. Myers, "Brasil, Reluctant Pursuit of the Nuclear Option", Orbis, Vol. 27, No 4, invierno 1984, p. 891.

IIbid, p. 892.

Dér Spiegel, julio 15 de 1985, p. 196.

sMyers, op. cit;, p. 892. 
bastante dudoso que el proceso de pitón a chorro sea competitivo con otras tecnologias de enriquecimiento tales como difusión gaseosa o centrifugado de gas.

La decisión de continuar construyendo la planta de enriquecimiento de Resende puede ser vista como una respuesta al éxito argentino para dominar el ciclo nuclear. A pesar de sus altos costos de producción y construcción se ha beneficiado de un status especial y no sufrió las severas restricciones económicas que afectaron a otras partes del programa nuclear. El complejo de Resende también contiene una planta para convertir el uranio enriquecido en elementos combustibles. Esta planta fue completada en 1982 y es capaz de producir 100 toneladas de elementos combustibles al año6.

La programada construcción de una planta de reprocesamiento ha sido pospuesta indefinidamente. Esto se debe principalmente a problemas financieros $\mathrm{y}$ a disputas sobre el lugar donde estará situada.

Con respecto a plantas de energía nuclear, los logros no son demasiado impresionantes. El acuerdo original preveía la construcción de ocho plantas (cada una con una capacidad de $1.245 \mathrm{Mw}$ ) para 1990, las que hubieran sido agregadas a la capacidad de 626 Mw del primer reactor. Este reactor, Angra I, en Angra do Reis en el estado de Río de Janeiro, fue comprado como un proyecto piloto de Westinghouse en 1972, y estaba programado para entrar en funcionamiento en 1977. Pero no empezó a generar electricidad hasta marzo de 1982 y fue forzado a cerrar poco tiempo después por las vibraciones en el generador, que sólo podía producir cerca de un 30 por ciento de su capacidad programada 7 . Angra I ya ha costado 18 mil millones de dólares y necesita aún inversiones de varios millones de dólares para funcionar correctamentes. Persisten serias dudas de que Angra I pueda alguna vez producir más de la mitad de su capacidad planificada.

Angra II y Angra IIr constituyeron los primeros dos reactores que fueron construidos bajo el acuerdo con la RFA por Kraftwerk Union (KwU). Angra II sólo estará listo en $199^{\circ}$ y Angra III todavía está en una fase muy temprana de construcción ${ }^{10}$, en cuya elaboración se encontraron muchas de las mismas dificultades que en Angra I debido a la carencia de substrato geológico sólido en la playa de Angra do Reis ${ }^{11}$.

${ }^{\theta}$ Nachrichten für den Aussenhandel, 18 de octubre de 1982.

TThe Latin American Times, marzo de 1983, p. 16.

${ }^{8}$ Latin American Regional Report, 9 de agosto de 1985, p. 7.

Der Spiegel, 15 de julio de 1985, p. 95; Latin American Regional Report, 9 de agosto de 1985, p. 7.

10Der Spiegel, 15 de julio de 1985, p. 95.

xpara mayores detalles sobre las dificultades técnicas encontradas durante la construcción de los reactores de Angra, compare Myers, op. cit., 898-895 
Un compromiso adicional incluido en el acuerdo era la formación de Nuclep, la empre:a conjunta que involucraba a Nuclebrás con firmas alemanas y austriacas para la manufactura de equipo pesado para reactores de energía. Nuclep comenzó a operar en 1980, después de que habían sido invertidos más de us $\$ 250$ millones en sus instalaciones en Itaguai, en el estado de Río de Janeiro. Debido a la marcada baja en la construcción de reactores, la planta está funcionando actualmente a un 30 por ciento de su capacidad ${ }^{12}$.

\section{El programa paralelo}

Aparte del programa nuclear oficial, existe una amplia variedad de proyectos de investigación nuclear dentro de un programa paralelo. De ésta, los proyectos principales se relacionan con la adquisición de tecnologías de reprocesamiento y enriquecimiento diferentes de aquellas incluidas en el trato con la RFA. Se empezaron en la misma época en la que el ritmo de la implementación del programa de 1975 se desaceleraba. Se les dio mayor importancia por el anuncio argentino del 18 de noviembre de 1983 de que había tenido éxito en la producción de uranio enriquecido ${ }^{13}$.

EI programa paralelo está protegido por SIPRON, el servicio especial de seguridad, quien está bajo la responsabilidad del $\operatorname{csN}^{14}$. A pesar del secreto oficial, sin embargo, frecuentemente ha habido informes de prensa sobre las actividades principales del programa15. Esto ha llevado a un cambio gradual en la política de prensa del gobierno, lo que al menos confirma la existencia de tal programa ${ }^{16}$.

El centro de investigación principal es el Instituto de Investigaciones Energéticas y Nucleares (IPEN), un instituto separado y financiado federalmente en la Universidad de São Paulo. Se está construyendo una planta de enriquecimiento de uranio, usando el método de gas centrifugado, con un programa conjunto de la Comisión de Energía Nuclear (CNEN) y la Marina. Se dice que también se es-

\footnotetext{
12Der Spiegel, 15 de julio de 1985, p. 95; Latin American Regional Report, 9 de agosto de 1985, p. 7 .

${ }^{13}$ Para mayores detalles sobre la reacción brasileña al anuncio argentino. comparar Leonard S. Spector, Nuclear Proliferation Today (Cambridge, Mass, 1984), pp. 255-63.

"Luiz Pinguelli Rosa, "Que fazer da Nuclebrás?", Revista Brasileira Tecnológica, Vol. 16, No 2, marzo-abril 1985, p. 62.

${ }^{15}$ Latin American Riegional Report, 5 de febrero de 1982; Der Spiegel, No 33, 1982, pp. 98-101; ISTOE; 19 de enero de 1983; O Estado de Säo Paulo, 9 de diciembre de 1983, p. 5; The Latin American Times, 30 de enero de 1984; pp. 2226; Folha de São Paulo, 11 de mayo de 1984 y 28 de abril de 1985; VEJA, Il de septiembre de 1985, pp. 65-66.

${ }^{10} \mathrm{~A}$ principios de 1983 Danilo Venturini, Secretario General del Consejo Nacional de Seguridad; anunció que el IPEN iba a continuar su proyecto para desarrollar la tecnología de enriquecimiento por centrifugado como parte de una
} 
taría trabajando en el enriquecimiento de uranio por rayos láser en el IPEN ${ }^{17}$.

Los expertos del IPEN han tenido exito en convertir el uranio natural a gas de uranio hexafluórico, que es el paso intermedio esencial para el proceso de enriquecimiento por gas centrifugado. En octubre de 1984 se abrió una pequeña planta de conversión ${ }^{18}$. Esto significa que una vez que la planta de enriquecimiento del IPEN esté operando, Brasil tendrá una capacidad de producir uranio altamente enriquecido en forma completamente autónoma y poco segura. También hay una unidad laboratorio de reprocesamiento en el IFEN, que es capaz de extraer pequeñas cantidades de plutonio del material irradiado o del combustible usado ${ }^{19}$.

En el mismo centro de investigación, la Armada está llevando a cabo un proyecto que apunta a la construcción de un reactor nuclear para ser usado para la propulsión de submarinos. Éste estará listo hacia 1992, a más tardar, de acuerdo al ex Ministro de Marina, Almirante $\mathrm{Da}$ Fonseca ${ }^{20}$. En el Laboratório de Estudos Avançados (LEA), en el Centro Técnico Aéreospacial (CTA) de la Fuerza Aérea, cerca de São José dos Gampos en el estado de São Paulo, se supone que se están llevando a cabo investigaciones en las tecnologías de enriquecimiento por rayo láser y de reactor de generación rápida ${ }^{21}$.

Inea alternativa al tratado brasileño-germano (Spector, op. cit., p. 251). El 18 de diciembre de 1983, el Ministro de Energla, César Cals, destacó la importancia de que el programa sin salvaguardas fuera llevado a cabo en el cra. Manifestó que la prioridad de su gobierno era "la conquista del átomo", significando el desarrollo del enriquecimiento y reprocesamiento usando tecnologias brasileñas. Temeroso de ser malinterpretado, él, y el ministro de relaciones exteriores más tarde, sacaron un comunicado reiterando el propósito pacifico de la tecnologla nuclear en Brasil (Spector, op. cit, p. 260); O Estado de São Paulo, 15 de diciembre de 1983. En octubre de 1984, el entonces presidente del cNEN, Rex Nazaré, confirmó la existencia de un "programa nuclear paralelo" como fue manifestado por el éxito de más de 100 nuevos productos para uso civil; Paulo S. Wrobel, "A polf́tica nuclear a Nova República", Perspectivas Internacionais, enero-febrero de 1985, p. 7. En el mismo mes, el Presidente Figueiredo hizo una visita muy publicitada al IPEN para observar una demostración del proceso de enriquecimiento por centrifugado, L. S. Spector, The New Nuclear Nations (New York/Toronto, 1985).

${ }^{27}$ Para detalles sobre la planta de enriquecimiento de gas centrifugado ver a Spector, op. cit. 251, y Spector (1985), op. cit., pp. 199-200.

${ }^{18}$ Spector (1985), op. cit. 199; para mayor información ver el detallado informe en Folha de Säo Paulo, 28 de abril de 1985.

${ }^{10}$ Esta capacidad fue públicamente anunciada por el Coronel Ernani Amorin, Director del IPEN, en junio de 1985. Ver O. S. Ferreira, "O Brasil no era nuclear", Politica e Estratégia, Vol. 2, No I, enero-marzo de 1984, p. 89.

so Folha de São Paulo, 28/4/85.

21 O Estado de São Paulo, 9/12/83. EI Centro Tecnológico do Exército (CENTEx) está llevando a cabo una amplia variedad de proyectos de investigación en la base de misiles de Marambai. El proyecto de investigación más importante estudia el uso de la energía nuclear para propulsión de satélites. Ver Myers, op. 
Estos proyectos son motivo de preocupación, ya que son realizados en secreto y cubren una amplia gama de tecnologías nucleares cuyo desarrollo no es vigilado y cuyo uso futuro puede ser militar. De acuerdo al informe de mayo de 1986 de la csN del Senado brasileño, es "probable que se estén haciendo amplias inversiones en el desarrollo de la tecnologia nuclear con fines militares"22.

\section{Bomba atómica}

En 1981 Ios ministerios de las Fuerzas Armadas emitieron una declaración conjunta en relación a que Brasil no estaba realizando ningún programa secreto para la construcción de una bomba atómica. Sin embargo, el senador Dirceu Cardozo, miembro del Comité de Investigación del Senado en el tratado nuclear brasileño con la RFA (CPI), declaró al mismo tiempo que no tenía dudas. de que todos los programas nucleares de Brasil estaban diseñados para adquirir la capacidad para construir la bomba ${ }^{23}$.

Gualquiera que sea la verdad del asunto, por ahora, Brasil carece de una fuente de uranio altamente enriquecido o de plutonio. Hay cuatro opciones diferentes para el país con respecto a la adquisición de estos materiales ${ }^{24}$ :

1. El método más directo sería usar las instalaciones de enriquecimiento basadas en la investigación actual en IPEN. Se espera que la unidad de centrifugado será completada en 1990. Esto sería lo más fácil para obtener material en cantidades suficientes para armas nucleares y tendría la ventaja de no ser controlada.

2. Una segunda posibilidad involucraría la unidad de laboratorio de reprocesamiento en el IPEN, extractando pequeñas cantidades de plutonio. La adquisición de vastas cantidades de combustibles no verificado sería el mayor problema en cuanto a lo que incumbe a esa opción.

3. La tercera posibilidad sería la producción de grandes cantidades de uranio altamente enriquecido bajo vigilancia. Esto sólo sería posible si la planta de enriquecimiento de Resende fuera usada. Ya que esta planta está diseñada para producir uranio enriquecido

cit., p. 896. CENTEX está siendo expandida y relocalizada. Se convertirá en el centro de la industria de armamentos de Brasil. Ver Latin American Regional Report, Brasil, 14/3/86, p. 6 .

${ }^{22} \mathrm{ABC}$, Madrid, 28/5/86, p. 43.

${ }^{2}$ Radio Bandeirantes, $19 / 6 / 81,2.30 \mathrm{GMT}$, como fue informado en MonitorDienst, Lateinamerika, 22/6/81, p. 4; el director de ventas de KwU, Gerlad Herzog, señala a principios de 1983 que era obvio que Brasil sería capaz de construir una botmba atómica "dentro de un año si sólo así lo deseara". Jornal do Brasil (JB), 25/4/88.

24Adaptado de Spector (1984), op. cit., pp. 898-899. 
de baja calidad, la IAEA podría reconocer inmediatamente un vuelco hacia la producción de material altamente enriquecido,

4. El método menos probable sería la obtención de una reserva de plutonio bajo vigilancia, puesto que esto incluiría el reprocesamiento del combustible usado en Angra I. Sin embargo, el permiso para reprocesar de los EE. UU., quien suministró la carga inicial, sería bastante improbable. El reprocesamiento de cargas posteriores, suministradas por Urenco, el consorcio de enriquecimiento de uranio británico-holandés-alemán, tampoco es probable, ya que los holandeses impusieron el requisito de que todo el plutonio extraído del combustible tenía que ser guardado bajo control multinacional. Incluso el uranio enriquecido por Brasil en Resende será mantenido bajo las regulaciones de IAEA, de acuerdo con el pacto tripartito con la RFA y el IAEA.

Desde que Argentina logxó completar el ciclo nuclear, oficiales militares brasileños importantes han hecho, varias declaraciones muy honestas sobre el propósito del esfuerzo de las investigaciones paralelas. Todas las declaraciones concordaban en que el objetivo era el dominio del ciclo del combustible nuclear, "permitiendo la fabricación de la bomba atómica"25; sólo diferfan en la fecha en que esto sería posible ${ }^{26}$.

En cuanto a lo que incumbe a la decisión política de adquirir armas nucleares, las declaraciones han sido ambiguas. Mientras que en 1985 el Brigadier Hugo de Oliveira Piva, director del cTA ${ }^{27}$ y el ministro de la Armada, General Leônidas Pires Gonçalves ${ }^{28}$ insissistieron en que tal decisión política aún no había sido tomada, en diciembre de 1983 el General Waldyr Vasconcellos, entonces Jefe del Mando General de las Fuerzas Armadas, rehusó confirmar o negar que esta decisión ya había sido tomada de hecho. Sólo dijo: "No podemos detener la investigación en el campo de la tecnología nuclear. Cualquier país que quiera progresar debe llevar a cabo tal investigación y quien logre dominar la tecnologia nuclear puede incluso fabricar una "bomba" 29 .

${ }^{20 \mathrm{E} l ~ G e n e r a l ~ d e ~ E j e ́ r c i t o, ~ L e o ̂ n i d a s ~ P i r e s ~ G o n c ̧ a l v e s ~ e n ~ d i c i e m b r e ~ d e ~} 1983$ en The Latin American Times, 30/1/84, p. 23. En el mismo sentido. el Brigadier Hugo de Oliveira Piva, Director del crA, en The Latin American Times, 30/1/84, p. 26, en diciembre de 1983; y el Ministro de Marina, Almirante Eduardo da Silva Fonseca Maximiano, citado en Spector, p. 261, en enero de 1984.

${ }^{2} \mathrm{El}$ Brigadier $\mathrm{H}$. de Oliveira Piva, Director del CTA, declaró en. agosto de 1985 que Brasil sería capaz de construir una bomba atómica dentro de los siguientes cinco años, Süddeutsche Zeitung, 20/8/85, p. 6, mientras que el ministro del Ejército, General L. Pires Gonçalves señaló a vEJA, $11 / 9 / 85$, p. 65, que

"la dominación del ciclo nuclear... será alcanzada al final de este siglo".

${ }^{27}$ Süddeutsche Zeitung; 20/8/85, p. 6 .

${ }^{2} \mathrm{VEJA}_{\mathrm{J}} \mathrm{II} / 9 / 85$, p. 65 .

${ }^{28}$ The Latin American Times, 30/1/84, p. 26. 
Hoy dia, un gran número de científicos nucleares brasileños aparecen obviamente involucrados en proyectos que están bajo control militar ${ }^{30}$ y que podrían construir los cimientos para un posible programa de armas nucleares. Tal programa dependería de una decisión polftica. A pesar de las declaraciones del Presidente Sarney en julio de 1985 respecto a que Brasil no tenía intención alguna de construir una bomba atómica y que no existía tal plan ${ }^{31}$, no quedan dudas de que Ios militares continúan empujando el programa paralelo hasta el punto donde se cumplan todas las precondiciones técnicas para la decisión política.

\subsection{Planes de energia nuclear}

La experiencia nuclear brasileña hasta 1975 estaba limitada a la experimentación con generadores de prueba y a las primeras etapas de construcción de Angra I, el reactor de Westinghouse. Sin embargo, con la firma del acuerdo de 1975 con la RFA, Brasil demostró formalmente que había decidido tomar un compromiso masivo con la energfa y tecnología nuclear. A un co to de 10 mil millones, Brasil esperaba estar produciendo $10.000 \mathrm{NW}$ de energía nuclear para 1990 , para complementar una generación de energ:a hidroeléctrica de $60.000 \mathrm{Mw}^{32}$. El Balance Nacional de Energia de 1978 proyectaba que en 1987 el 4,6 por ciento del consumo de energía del Brasil sería obtenido por energía nuclear ${ }^{33}$. Más aún el gobierno brasileño esperaba desarrollar una industria competitiva de construcción e ingeniería de reactores nucleares para proveer los mercados nacionales e internacionales.

A fines de 1978 el presidente de Nuclebrás (la empresa estatal responsable de la implementación del programa), Nogueira Batista, predijo que el costo total del programa llegaría a los 15 mil millones de dólares, a pesar de que los críticos ya habían aseverado que costaría el doble de esa cifra ${ }^{34}$. En 1983, el cPI del Senado concluyó que la realización del programa para el año 2000 llegarfa a un costo total de $\$ 24.800$ millones a 31 mil millones ${ }^{35}$.

En 1982 Nuclebrás sufrió una reducción de 40 por ciento en su presupuesto ${ }^{36}$, y el ambicioso programa nuclear brasileño se frenó

\footnotetext{
${ }^{30}$ Los cientificos que trabajaron en IPEN con la armada aparentemente reciben dos salarios. Folha de Sã̃o Paulo, 28/4/85.

Die Tageszeitung, $4 / 7 / 85$.

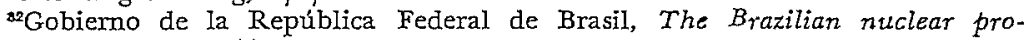
gramme (Brasilia: 1977), p. 10.

\$2 para proyecciones detalladas, Rep. de Brasil, National Energy Balance 1978 (Brasilia: 1978).

"William H. Courtney, "Nuclear choices for friendly rivals", en J. A. Yager. (ed.), Non-proliferation and u.s. Foreign Policy (Washington D. C.: 1980), p. 245.

${ }^{35} \mathrm{CPI}$, p. 71 ; expertos independientes estimaron el costo total en 36 mil millones de dólares. Ver IsTOE, 19/1/83.

${ }^{80}$ Myers, op. cit., p. 894.
} 
a un ritmo más realista. La fecha original para la completación de las ocho plantas nucleares fue pospuesta de 1990 al año 2000. Se continuó la construcción de Angra Ir; el término de la construcción de Angra IIr fue retrasado hasta 1990; y en Iguape, en el estado de São Paulo, sólo se completó la adquisición de los terrenos para la construcción de los siguientes dos reactores.

La posición exacta del nuevo gobierno de Sarney con respecto a las políticas nucleares aún no está definida. Pero la necesidad de

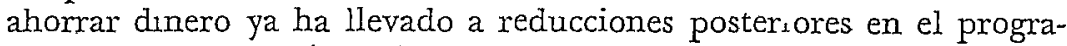
ma de energía nuclear. Los primeros grandes pa:os del gobierno para reducir el déficit fiscal de alrededor de 18.200 millones de dólares ${ }^{37}$ golpearon muy fuerte al programa nuclear. Sólo se le permitió continuar a Angra II. Nuclebrás, que, según su nuevo presidente Licinio Seabra, ha acumulado una deuda de $\$ 2.400$ millones, sufrió un corte de unos $\$ 117$ millones $^{38}$. Seabra consideró que los fondos que quedaban eran suficientes para completar Angra II y para mantener una actividad mínima de búsqueda y enriquecimiento de uranio así como para hacer algunos progreso: en Angra IIr, con el fin de no violar algunos contratos ${ }^{38}$. Aún se debe tomar una decisión final con respecto a la complementación de Angra Irr.

En su primer discurso of cial respecto a este tema, en julio de 1985, el presidente José Sarney reiteró el interés de Brasil en dominar la tecnología nuclear con fines pacíficos. Pero al mismo tiempo dejó en claro que las dificultades financieras necesitaban "algunas correcciones estratégicas" del programa ${ }^{40}$. Dos semanas después, el Ministro de Minas $y$ Energía, Aureliano Chavez (quien era vicepresídente bajo Figueiredo y quien aspira a ser el próximo presidente), se declaró a favor del programa de energía nuclear original y de terminar el trabajo en Angra im, a pesar de los costos cercanos a los $\$ 10$ millones. Subrayó que la adquisición por parte de Brasil de esta tecnología compensaría el sacrificio involucrado ${ }^{41}$.

Como resultado de la desesperada situación financiera en Brasil, no habrá trabajos posteriores en la construcción de reactores de energ'a nuclear dentro de los próximos dos años, excepto por aqueIlos ya en construcción; e:to podrfa no incluir a Angra III.

\section{Desarrollo de la toma de decisiones nucleares}

\subsection{Evolnución histórica}

En el desarrollo de los programas nucleares brasileños se pueden

${ }^{3}$ Latin American Weekly Report, 12/7/85, p. 4.

${ }^{28} O$ Estado de São Paulo, $15 / 5 / 85$.

"Tbid., 1/8/85, p. 28.

10Die Tageszeitung, 4/7/85.

${ }^{4} O$ Estado de São Paulo, 17/7/85, p. 22. 
distinguir cuatro fases. La primera, la "fase independiente", empezó en 1945 y duró hasta 1953. La segunda fase, desde 1954 a 1966, estuvo caracterizada por una estrecha cooperación con los Estados Unidos. Durante la tercera, la "fase activa", de 1967 a 1978, Brasil diversificó sus socios en un intento por construir una industria y una infraestructura tecnológica nuclear. La cuarta fase coincidió con la apertura, el proceso de liberalización política en el pais, y viene desde 1979 .

Debido a la crítica abierta al programa y al incremento de las dificultades financieras, esta cuarta fase consistió en un proceso progresivo de revisión del programa de energía atómica.

\section{La fase independiente (1945-1953)}

La fase independiente se caracterizó por el intento de mantener el ritmo a un nivel científico con los rápidos avances tecnológicos en el campo nuclear. El 10 de julio de 1945 se concluyó el primer tratado brasileño para cooperación nuclear con Estados Unidos ${ }^{42}$. EI tratado, que se mantuvo en secreto, incluía el abastecimiento anual de 5.000 toneladas de monacita, un mineral que contiene uranio. En 1951 se fundó el Consejo Nacional de Investigaciones (CNPq), lo que institucionalizó las investigaciones básicas. Su objetivo fue usar las reservas brasileñas de torio y uranio para el desarrollo de tecnologías nucleares. A continuación, en 1953, Brasil trató de adquirir tres centrífugas de gas para enriquecimiento de uranio por medio de un trato secreto con la RFA. Como resultado de la intervención norteamericana, oficiales de ocupación británicos embargaron estas centrífugas justo antes de su embarque ${ }^{43}$. De esta forma, el primer intento de Brasil por recibir equipamiento atómico, falló.

\section{Cooperación con los Estados Unidos (1954-1966)}

En 1954 se fundó la Comisión Nacional de Energía Nuclear (CNEN), marcando el fin de la fase independiente ${ }^{44}$. Después de que el programa "Atomos para la paz", de los Estados Unidos, puso información cientifica no clasificada a disposición de muchas naciones, Brasil concluyó un tratado con los Estados Unidos que incluía el entrenamiento de personal y la entrega de tres reactores de investigación. Éstos fueron decisivos en 1957 (São Paulo), 1960 (Belo Horizonte), y 1965 (Rio de Janeiro).

En 1956 el Consejo Nacional de Seguridad aprobó el reemplazo

22O. Guilherme, O Brasil e a era atómica (R. de J.: 1957), p. 85.

${ }^{4}$ V. L. Johnson, "Brazilian Nuclear Development: Intent and Capabilities", Doctoral Thesis, U. de Michigan, 1979, p. 72.

*R. M. Schneider, Brazil: Foreign Policy of a Futıre World Poiver (Boulder, Colorado: I976), p. 49. 
del CNPq con el CNEN y el Fondo Nacional de Energía. Todas las políticas nucleares nacionales empezaron a ser coordinadas por el CNEN, que e:taba bajo la jurisdicción del Ministerio de Minas y Energía.

Durante esta segunda fase se usaron principalmente dos argumentos para justificar el interés brasileño en la tecnología nuclear: la creciente demanda por energía debido al crecimiento económico proyectado, y el posible uso de PNEs para programas de desarrollo más grandes. Más tarde, con la aparición de Francia y China como nuevas potencias nucleares, el status empezó a jugar su papel.

Se hicieron progresos en el área nuclear durante los 60s. Durante el gobierno del presidente Juscelino Kubitschek (1956-1961), quien había cortado relaciones con Ios Estados Unidos, se favoreció la construcción de una industria nuclear basada en el modelo norteamericano con sus reactores de agua liviana (usando uranio enriquecido). El presidente Jânio Quadros (196I; elegido alcalde de São Paulo en 1985) y el presidente João Goulart (1961-1964) favorecieron una polftica exterior independiente y por lo tanto optaron por Ios reactores franceses de agua pesada por su dependencia del uranio natural. Los ractores de agua liviana dependen de una provisión de uranio enriquecido, que era en esa época monopolio de Ios Estados Unidos.

La nueva cooperación científica con Francia fue interrumpida por el golpe militar de 1964. Los oficiales pro-norteamericanos quebraron las negociaciones con Francia por un reactor de agua pesada y colocaron una moratoria a todas las negociaciones para la transferencia de tecnología nuclear a Brasil; esto persistio durante los primeros años del gobierno militar ${ }^{45}$. Desde 1964, el $\operatorname{csN}^{\text {, }}$ que está compuesto casi exclusivamente de oficiales militares, ha sido el cuerpo que toma las decisiones. Esto ha llevado a un alejamiento dê las consideraciones cientfficas y hacia motivaciones económicás y militares para la adguisición de tecnología nuclear.

\section{La fase activa (1967-1978)}

La tercera - y más activa - fase del programa nuclear brasileño empezó en 1967. El csN declaró que una política nuclear independiente era vital para el desarrollo del país. Así, esto dio al programa nuclear una prioridad de largo plazo dentro de la "doctrina de seguridad nacional" de los militares, que era la base del plan de desarrollo brasileño a partir de $1964^{46}$.

Hubo varias razones para esta decisión. Una era que el nuevo gobierno del Presidente General Artur da Costa e Silva (1967-69)

wohnson, (1979), op. cit., p. 72.

uschneider, op. cit., p. 91. 
necesitaba un nuevo lema nacional para su periodo y estaba bus: cando una conexión entre la persecución de un programa nuclear. nacional y el desarrollo económico. Otra razón fue que con. la conclusión del Tratado de TIatelolco en 1967 y del NPT en 1968, los militares brasileños temían que se le impidiera a Brasil ganar más status e independencia a través de su propio programa.nuclear. De esta forma, Brasil ratificó el TT sin descartar el Art. 28, I y no firmó el NPT por su supuesta discriminación entre estados con y sin armas nucleares. Una tercera razón para esta posición era la esperanza que tenf́a el gobierno de ganar el apoyo interno en vista de la favorable actitud de la oposición hacia las políticas de corte nacionalista.

Así, en el año 1967, la Comisión Nacional de Energía Nuclear recomendó que Brasil debía continuar con sus planes para la instalación de un generador de energía atómica. Sostenía firmemente que se debía seguir la política de adquirir tecnología atómica desde el extranjero, pero también apoyaba el plan para una eventual producción de combustibles atómicos brasileños ${ }^{47}$. El GNEN estimuló investigaciones posteriores, pero la tarea práctica de supervisar el desarrollo nuclear acelerado fue realizada por la Compañía Brasileña de Tecnología Nuclear (CBTN), que fue creada en 1971.

A su vez, la CBTN fue transformada, en 1974, en Nuclebrás. Fundada como contraparte de Petrobrás, el monopolio nacional de petróleo, Nuclebrás básicamente ejerció el control monopólico sobre el desarrollo nuclear nacional. Sin embargo se permitieron los acuerdos conjuntos con inversores menores extranjeros en la construcción de reactores, la explotación de uranio y. la investigación. ${ }^{48}$.

La CBTN formalizó el contrato con Westinghouse para la construcción del primer reactor de energía nuclear del Brasil, Angra I, en 1972. Al mismo tiempo, se buscaba la cooperación nuclear con Canadá, la RFA, Francia, India e Israel. En 1974, cuando la Comisión de Energía Atómica de EE. UU. (AEC) se negó a garantizar la entrega de uranio enriquecido para los proyectados reactores de agua liviana, el apoyo para los Estados Unidos como el principal proveedor nuclear empezó a erosionarse rápidamente ${ }^{49}$. En consecuencia, las negociaciones con la RFA se intensificaron, lo que llevó à la firma del acuerdo de junio de 1975.

Como resultado de este acuerdo, el Gobierno decidió desmaritelar la mayoría de los equipos de experimentación que operaban en campos fuera de aquellos favorecidos por el trato con la RFA. Siguiendo las proposiciones de Nuclebrás, que lideraba el grupo de

"Tohnson (1979), op. cit., p. 73.

spara mayores detalles sobre inversiones extranjeras en desarrollo nuclear hasta el '75, Ted Johnson (1979), ap. cit., pp. 74-77.

4Schneider, op. cit., p. 92. 
-presión en favor del trato .con la RFA, el Instituto Militar de Ingenieros (IME), manejado por el ejército, cesó su experimentación para la producción de agua pesada, y el Instituto de Ingeniería Nuclear (IEN), que llevaba a cabo investigaciones de generadores rápidos, fue cerradoso.

El programa fue vigorosamente implementado por el enérgico presidente de Nuclebrás, Paulo Nogueira Batista, quien lideraba al impresionante número de 6.000 burócratas y técnicos empleados por Nuclebrás y sus firmas subsidiarias ${ }^{51}$. Era un diplomático y economista que siempre tenía acceso al presidente, general Ernesto Geisel (1974-79).

El programa nuclear se convirtió en la pieza central del período del Presidente Geisel. Esta prominencia expuso al programa a una creciente critica, aún durante la presidencia de Geisel. Era considerado como demasiado ambicioso y demasiado caro. Sin embargo, la censura limitaba severamente las criticas abiertas ${ }^{52}$.

La fase revisionaria (1979.)

La cuarta fase empezó con la inauguración del Presidente Joâo Batista Oliveira de Figueiredo (1979-85). Esta fase revisionaria actual está caracterizada por un desaceleramiento radical de la implantación del programa nuclear de 1975 y un cambio de recursos hacia Ios esfuerzos de investigación más local y lejos de la transferencia masiva de tecnología.

A principios de 1978 el programa de enriquecimiento ultracen. trifugado en el IPEN fue iniciado secretamente en una cumbre federal que incluyó a representantes del Servicio Nacional de Inteligencia (SNI), la Comisión Nacional de Energía Nuclear y Nuclebrás. E1 desarrollo de instalaciones alternativas de enriquecimiento fue declarado parte del esfuerzo de seguridad nacional ${ }^{53}$.

A continuación, el cSN argumentó que Brasil debía haber continuado su investigación de la tecnologia de agua pesada, ya que era mucho más fácil obtener plutonio de graảo para armas del desecho de un reactor de uranio natural que de uno de uranio enriquecido ${ }^{54}$. Se organizaron varios proyectos nuevos fuera de la jurirdicción de Alemania Federalos:

1) El cNEN, en colaboración con Francia e Italia, comenzó a construir un nuevo gran centro de investigación en Campo di Roma, di-

${ }^{\circ}$ Latin American Regional Report, 5/2/82.

'ISTOE, 19/1/83.

${ }^{83}$ K. R. Mirow, Das Atom Geschaft mit Brazilien; Ein Milliardenfiasko (Frankfurt/N. Y.: 1980), pp. I43-6.

raspector (1984), op, cit., p. 251.

*Latin American Regional Report, 5/2/82.

soIbid. 
señado para realizar estudios en la tecnología de los generadores rápidos.

2) La Fuerza Aérea completó su gigantesco Laboratorio de Estudos Avançados en el cTA, diseñado exclusivamente para llevar a cabo investigación nuclear.

3) El ejército organizó su centro de investigación, Gentex, con una alta proporción de sus recursos dedicados a la investigación nuclear.

4) IPEN se expandió rápidamente. Se aceleraron los trabajos de construcción de su planta piloto para la producción de uranio hexafluorado.

Los militares empezaron a ejercer control sobre los proyectos más importantes en IPEN. En 1983, el presidente Figueiredo designó al coronel Durvaldo Gonçalves, por largo tiempo sustentador del desarrollo de armas nucleares, como nuevo director del IPEN. Él había sido transferido de la responsabilidad del Estado de São Paulo a la supervigilancia federal, después de la victoria del entọnces opositor Partido do Movimento Democrático Brasileiro (PMDB) en las elecciones estaduales de fines de 1982. Bajo el gobierno de Sarney, se devolvió el IPEN al control del Estado en 1985, pero la marina ahora controla los proyectos de investigación más sensitivos bajo un sistema de administración dual56.

El programa de energía nuclear estaba considerado por la oposición como un símbolo de las prácticas "faraónicas" seguidas por los gobiernos militares brasileños por muchos años. Se estaba desarrollando un sentimiento generalizado de que Brasil no podía afrontar los gastos planificados de miles de millones de dólares en energía nuclear que de otro modo hubieran podido ser usados en aliviar necesidades sociales imperiosas; en 1979 el Gobierno enfrentaba una deuda pública de 50 mil millones, que se habían duplicado hacia fines dèl periodo de Figueiredo.

La crítica más fuerte y especifica provino de la comunidad científica brasileña, que no había estado involucrada en el programa nuclear de 1975. Sus puntos de vista tuvieron creciente impacto en la opinión pública. La prestigiosa Asociación Brasileña de Científicos (SBPC) ya había criticado en 1975 el hecho de que los expertos nucleares brasileños más relevantes no hubieran estado involucrados en la conclusión del acuerdo con la RFA ${ }^{57}$. Establecieron que los distintos grupos de investigaciones nucleares aún no habían decidido sobre la tecnología nuclear apropiada para Brasil. Más aún, se

${ }^{6}$ Folha de São Paulo, 28/4/85. pág. 9.

"Ver. J. Leite López "Nuclear Energy in Brazil", sin publicar, Munich, 8/77, 
suponía que no habian sido consultados precisamente por esta decisión pendiente entre el uso del uranio natural y el uso de uranio enriquecido.

Posteriormente, científicos sucleares prominentes como José Goldemberg, David N. Simon, y Luiz Pinguelli Rosa, publicaron extensamente sus críticas al programa de energía nuclear ${ }^{58}$. Su principal queja era que la importación de equipo nuclear alemán no daba oportunidad a los científicos locales de desarrollar sus propias habilidades. Joaquim de Carvalho, ex director para el desarrollo industrial de Nuclen, la subsidiaria de ingeniería de Nuclebrás, y Pinguelli.59 expresaron sus dudas acerca de que realmente se llevara a cabo la transferencia de tecnología. Carvalho ${ }^{60}$ criticaba los programas de transferencias por ser "de hecho sólo servicios para asis. tencia técnica ligada con la venta de patentes y licencias".

Más aún, los científicos subrayaron que el crecimiento de la démanda de electricidad había sido calculado en exceso por el gobierno y que las potencialidades de ahorro de energía y de fuentes alternativas de energía no se habian tomado en cuenta ${ }^{61}$.

Para demostrar su compromiso con la apertura, el gobierno de Figueiredo permitió a los medios de comunicación que cubrieran la implementación del acuerdo nuclear extensivamente. EI gran número de dificultades técnicas y los retrasos postexiores, los costos en constante alza y la corrupción generalizada erosionaron no sólo la imagen pública del acuerdo tan celebrado sino también del régimen militar en sí mismo. Uno de los escándalos principales fue el de la firma brasileña Norberto Odebrecht, a quien se le confiaron los trabajos de ingeniería civil de Angra II y Angra III a pesar de que no se había llamado a propuesta.

Las audiencias ante el Comité Investigador del Senado en 1978 ayudaron en forma especial a dar Iuces sobre las "irregularidades" en la implementación del programa nuclear ${ }^{62}$. Tanto miembros del Congreso como industriales usaron el tema en sus luchas personales

svex C. A. Girotti, Estado nuclear no Brasil (São Paulo: 1984), pp. 197-206; J. de Carvalho, Energia e Meio Ambiente (R. de J.: 1980); y D. N. Simon, Carvalho, Goldemberg, Menezes, Pinguelli Rosa y Gomes de Oliveira, Energia Nuclear en Questao (R. de J.: 1981).

${ }^{\circ}$ Pinguelli Rosa concluyó que los técnicos brasileños enviados a la RFA no estaban adquiriendo un conocimiento básico ni tenfan una idea general sobre el marco en el cual se desarrollaba su trabajo IsTOE, 19/1/83.

${ }^{\infty}$ Carvalho (1980), op. cit., p. 73.

${ }^{\infty}$ Girotti, op. cit., pp. 136-7; Carvalho (1980), op. cit., pp. 36-4I y 70; sobre energía solar y biomasa comparar M. Duayer, Ph. Gummett y K. Green, "The Brazilian Nuclear Power Programme: A Case not Proven", Energy Policy, Vol. 9, No 4, dic. 1981, pp. 323-6.

"H. Krugmann, "The German-Brazilian Nuclear Deal", The Bulletin of Atomic Scientists, Vol, 37, No 2, feb. 1981, p. 32; para detalles sobre las posturas de los diferentes partidos políticos comparar Girotti, op. cit., pp. 206-213. 
contra los opositores políticos y/o comerciales. Se debe recalcar, sin embargo, de que no hubo en ningún momento un significativo movimiento antinuclear. Para citar a Girotti63, "el movimiento antinuclear brasileño es más conocido en Europa que en su propio pais".

La dependencia de Brasil en la tecnología externa también recibió cada vez más críticas. El amplio apoyo original para el gobierno de Geisel por no haberse inclinado ante las pres:ones norteamericanas se erosionó rápidamente. Carvalho ${ }^{64}$ expresó un punto de vista cada vez más popular al hacer notar que la tradicional dependencia de los Estados Unidos sólo había sido camb:ada por una nueva dependencia con la RFA.

Desde que el gobierno de Sarney asumió, la comunidad cientifica ha incrementado aún más su influencia. Esto ha sido demostrado más claramente por la designación de un conocido científico nuclear, crítico del programa de 1975, el profesor José Goldemberg, a la presidencia de la Compañía de Electricidad del Estado de São Paulo (CESP). Era uno de los asesores claves en el campo de la energía del candidato presidencial Tancredo Neves. Goldemberg ejer. ció una influencia importante en las recomendaciones adoptadas en Ja Reunión Anual de 1985 del SBPC sobre energía nuclear ${ }^{65}$. Estos inclufan:

1) Ia disolución de las empresas conjuntas de Nuclebrás con kwu;

2) la creación de nuevas oporturidades de producción para las subsidiarias de Nuclebrás;

5) la reestructuración de la investigación nuclear (estrictamente para fine pacíficos), uniendo al CNEN y a todos los institutos de investigación nuclear con las universidades y la transferencia del CNEN a la jurisdicción del Ministerio de Ciencia y Tecnología;

4) la evaluación de las necesidades de largo plazo con un sentido cientffico y con la participación de d:ferentes sectores de la sociedad.

En esta reunión, los ejecutivos de Nuclebrás sostuvieron, por primera vez, discusiones con científicos que se oponían al trato con la RFA ${ }^{66}$. En agosto de 1985 se creó un comité del gobierno para la revisión del programa nuclear, que entregó su informe en abril de 198667. El comité, "creado bajo el auspicio del Ministerio de Minas y Energía, incluía a miembros de la; compañías estatales Nucle-

"Girotti, op. cit., p. 213.

"Carvalho (1980), op. cit., pp. 98-99.

${ }^{\infty}$ O Estado de São Paulo, $17 / 7 / 85$, p. 22.

"Latin American Regional Report, 9/8/85.

'Latin American Weekly Report, 25/4/86, p. 3; Nachritchten für den Aussenhandet, $15 / 5 / 86$. 
brás y Gesp, de varios ministerios (Minas y Energfa, Ciencia y Tecnología, Industria y Comercio), del CNEN, y de la Asociación Brasileña de Científicos.

El comité recomendó la completación de Angra Ir y Angra iri en 1992 y 1995 respectivamente, y que las decisiones sobre la construcción de otras plantas rucleares no debían zer tomadas antes de 1989. Sin embargo, recalcaba que hacia el año 2015 no habría más posibilidades de incrementar la producción de energía hidroeléctrica.

El comité recomendó también la reactivación de la minería y molienda de uranio en Poços de Caldas. Con respecto a la planta de enriquecimiento por pitón de chorro en Resende, los miembros del comité favorecieron el término de la primera cascada, y recomendaron que la decisión sobre la completación de la planta a escala industrial fuera pospuesta hasta 1989. Con respecto a la planta de reprocesamiento el comité no vio ninguna necesidad inmediata de construirla. El costo total de completar los proyectos del programa nuclear actualmente bajo consrucción está estimado en $37.800 \mathrm{mi}$ llones de dólares. El comité también propuso que las subsidiarias de Nuclebrás: Nuclam, Nuclei, Nuc'ep (ver No 81), fueran integradas a Nuclebrás, quien a su vez debiera ser privatizada, y que Nuclen debiera formar parte de Electrobrás8. Finalmente el comité recomendó que la cooperación nuclear con la RFA fuera continuada, pero que el acuerdo de 1975 fuera adaptado al "nuevo ritmo y estructura" del programa de energía nuclear brasileño. La ausencia de miembros del cNS, que hasta entonces habian ejercitado una influencia determinante en la política nụclear brasileña, era notoria. Pero esta ausencia no debe ser interpretada como una pérdida de la influencia militar. Por el contrario, los militares permanecen firmemente en control de la mayor parte del programa paralelo e indudablemente han ejercido su, influencia en las decisiones de este comité a través de sus propios canales.

Desde principios de los 80 s, los intereses de la comunidad cientifica, el grupo de presión de la hidroelectricidad y los militares coinciden en presionar por mayores investigaciones nucleares locales y por una reducción del programa nuclear de 1975 a sus niveles más bajos posibles, excepto por la explotación y enriquecimiento de uranio. Los militares permanecerán como un importante factor en el proceso de toma de decisiones de la política nuclear. Parece que han hecho de su control sobre la tecnologia nuclear, el sector energético, Ia industria de armamentos y las actividades aeroespaciales,

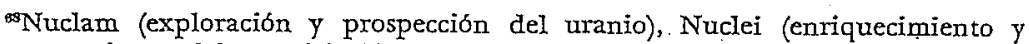
reprocesamiento del uranio), Nuclep (componentes pesados), Nuclean (ingeniería). 
Wolf Grabendorff / La politica nuclear y de no-proliferación de Brasil

uná condición importante para su retiro del ejercicio del poder en Bràsil. :

\subsection{Hidraelectricidad y absorción inter-agencias}

A través de todas estas fáses de la política nuclear brasileña hà habido una fuerte rivalidad entre los ministerios y otras agencias involucradas, lo cual ha producido un altó nivel de "absorción interagencias" (Johnson) ${ }^{69}$ que ha tenido importantes repercusiones eṇ Ia formulación e jmplementación de los programas nucleares.

E to ha sido más obvio desde 1974 en adelante. Pocas personas pàrticiparon en las negociaciones con la RFA, que empezaron en máyo de 1974 y fueron conducidas principalmente por el Secretario Géneral del csN, con consejos técnicos del Ministerio de Minas.y Energía y, consejo legal dèl Ministerio del:Exterior ${ }^{70}$. Más tarde; e? senadór Tavora participó como representante del Congreso ${ }^{71}$. Pero no había ningún representante ni del CNEN ni-del Ministerio de Comercio e Industria. El gobierno brasileño-justificó más tarde este secreto al referirse a las presiones foráneas - aludiendo indirectamente a jos EE. UU.- que impidieron negociaciones abiertas.

, La ta principal disputa inter-agencias siempre ha sido aquella en que unos favorecen la hidroelectricidad y otros favorecen la energía nuclear, comprometiendo directamente a dos agencias estatales, Elečtrobrás y Nuclebrás ${ }^{72}$... Ambas agencias usaron criterios diferentes para estimar los costos: de las plantas de energía nuclear y la cậntidad de energía generada por ellas. Nuclebrás estimó en 1976 que el potencial hidroeléctrico era de unos $150.000 \mathrm{MW}$, con un crecimiento anual de demanda de electricidad de un 10 por ciento, mientras que en 1980 Electrobrás calculó las reservas hidroeléctricăs en 213.000 $\mathrm{Mw}$ y el crécimiento anual de demanda de energía en un 6 por ciento ${ }^{73}$. Es obvio que la necesidad de energía nuclear es mucho más alta de acuerdo a la primera de estas estimaciones que a la siegunda. Más aún, las estimaciones de Nuclebrás predijeron que Ia energía nuclear sería necesaria para la década de los $90 \mathrm{~s}$, mientras que Electrobrás consideró que las fuentes adicionales de energía sólo sérían necesarias para el año 2020.

$-\quad$ in

"Johnson (1979), op. cit., p. 78.

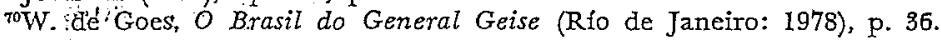

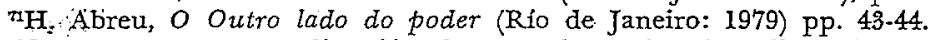

reomparar para una discusión de esta alternativa Carvalho (1980), op. cit., .p 70 a 9;: Girotti, op. cit., pp. 134-6;. Mirow, op. cit., pp. 136-42, A. Filkenstein "Brazil, the U.s. and Nuclear Nonproliferation; American Foreign Policy at the Crossroad". The Fletcher Forum, Vol. 7, No 2, verano 1983, pp. 301-2.

${ }^{7}$ L. C: Meneses y D. N. Simon "Dois Erros em cadeia: A politica nuclear e a estrutura organizacional do Programa Nuclear Brasileiro", en D. N. Simon et. al. (eds.), Energía Nuclear en Questao (R. de J.: 1981), pp. 27-9. Para com- 
Las cifras de Electrobrás Ilevaron à César Cals, entonces ministro de Minas y Energía, a hacer el excepcional comentario, en agosto de 1981, de que "la instalación de reactores nucleares no responde al criterio de energía sino al criterio de absorción de tecnología"74. Esto implica que Ios generadores de energía que serían adquiridos como parte del trato con la RFA en un paquete nuclear fueron percibidos como innecesarios para la producción de energía aún por el ministro responsable.

Basándose en las mismas suposiciones, Goldemberg, el presidente del cESP, propuso en julio de 1985 que el programa nuclear debía ser reducido en favor del desarrollo futuro de la energía hidroeléctrica ${ }^{75}$. Esta fuente de energía es considerada por él y por una parte importante de la comunidad científica como más barata ${ }^{76}$, técnicamente más fácil y con menores riesgos para el medio ambiente. Más aún, ven el potencial, hidroeléctrico como suficiente para la demanda de energía brasileña hasta bien entrado el próximo siglo.

\subsection{Influencias externas}

Brasil ha firmado tratados de cooperación nucleár con muchos pafses, incluyendo Algeria, Argentina, Bolivia, Chile, Colombia, Ecuador, Francia, RFA, Iraq, Israel (una convención), Italia, Paraguay, República Popular de China, Perú, Portugal, España, Suiza, el Reino Unido, Uruguay, Ios EE. UU., Venezuela y la Comunidad Europea de Energía Atómica ${ }^{77}$. Pero una influencia determinante en las politicas nucleares brasileñas ha sido ejercida directa e indirectamente sólo por sus dos socios económicos más importantes, los Estados Un'dos y la RFA. Ellos son los principales acreedores, inversionistas y socios comerciales de Brasil. El involucramiento de empresas norteamericanas y alemanas del Este en el desarrollo económico de Brasil y la cooperación científica con ambos países crearon la infraestructura económica y científica necesaria para la cooperación nuclear posterior.

br

parar: el potencial hidroeléctrico de Europa Occidental totaliza $158.000 \mathrm{MW}$, ver K. P. Erickson, "State Entreprenourship, Energy and the Political Order in Brazil", en: T. C. Bruneau y P. Faucher (eds.), Authoritarian Capitalism: Brazil's Contemporary Economic and Political Development (Boulder, Col.: 1981), p. 149 .

${ }^{74}$ Citado en Menezes y Simon, op. cit.

${ }^{T} O$ Estado de Säo Paulo, 17/7/85, p. 22.

${ }^{76}$ Para detalles de la comparación de costos ver Girotti, op. cit., p. 133; L. Pinguelli Rosa, "O Papel da Energfa Nuclear na Geraçao de Energfa Elecrica no Brasil", en Simon et. al.; op. cit., pp. 42-47.

"Spector 1984, op. cit., p. 252: para detalles sobre acuerdos con Colombia, Chile y Venezuela ver J. R. Reddick, "The Tlatelolco Regime and Nonproliferation in Latin America", Ințernational Organization, Vol. 35, No I, invierno 1981, pp. 127-8; el tratado de cooperación con la Comunidad Europea de Energła Atómica expiró en junio de 1985. 
Con la decisión de Brasil de comprar un generador a Westinghouse en 1972, les Ex. Uv. tenían una posición líder como socios nucleares de Brasil. Hubo, sin embargo, tres factores que llevaron a un deterioro de esta relación:

1) la creciente crítica en Brasil sobre la dependencia en la UP;

2) la decisión de 1974 tomada por la Comisión dé Energía Atómica (AEC) de los EE. UU. de no renovar su garantfa para el aprovisionamiento de uranio enriquecido;

3) Ia política de no-proliferación de los EE. Uu., más severa después de la explosión nuclear de 1974 en India.

Deșde una perspectiva brasileña, la RFA aparecía como un socio mucho más àsequible que los Estados Unidos porque combinaba poderes económicos y tecnológicos similares con una inhabilidad $-\mathrm{y}$ desgano- para aplicar presiones políticas: La firma del acuerdo fue celebrada, de este modo, como un hito: Brasil se había salido de la cooperación con la dominante Norteamérica y se había encaminado hacia un nuevo modelo de asociación entre el Primer y el Tercer Mundo.

Desde 1975 a 1977 los gobiernos de Ford y Carter aplicaron una creciente presión para alcanzar una cancelación o al menos una revisión del tratado brasileño con Alemania Federal, enfocando el asunto en el aprovisionamiento de instalaciones para enriquecimiento $y$ reprocesamiento. Los funcionarios alemanes respondieron declarando que las protestas de Washington no surgian de preocupaciones altruistas sino de un egoista interés comercial con el gobierno norteamericano interviniendo en favor de su frustrada industria ${ }^{78:}$ Cuando el presidente Carter combinó sus esfuerzos de noproliferaćón con grandes iniciativas de derechos humanos y'con un cambio en la postura de los Estados Unidos en relación con los sưministros de armas, las relaciones brasileño-norteamericanas alcanzaron uno de sus puntos más bajos en el perfodo de postguerra ${ }^{79}$.

A pesar de las múltiples presiones externas y constreñimientos dentro de la relación triangular Brasil-RFA-Estados Unidos, Brasil se las ha arreglado para jugar un rol importante en ella. La estrategia brasileña ha consistido principalmente en tomar ventaja de la compe-

Tơilkenstein, op. cit., p. 282; Girotti, op. cit., pp. 82-84. Este argumento se vio reforzado por el envio de un representante de la us. Bechtel Corporatión a Brasil en marzo de 1975, para discutir la construcción de una planta de enriquecimiento.

${ }^{78 R}$. Roett $\Varangle$ G. Wiegand, "The Role of Argentina and Brazil: A View from the United States", Occ. Paper de sars Center of Brazilian Studies, Washington D. C., 1985, p. 9; Brasil renunció en 1977 a'la ayuda militar de 25 años con los Estados Unidos en medio de la fuerte condena por la interferencia de Estados Unidos en asuntos brasileños. 
tencia y las diferencias entre los Estados Unidos $y$ la RFA, fortaleciendo de este modo su propia.posición. El temor de Alemania Federal de perder el mercado brasileño a otros proveedores aumentó la influencia de Brasil sobre la RFA, particularmente después de la debacle del acuerdo de 1979 entre Irán y la RFA.

Desde que Ronald Reagan asumió la presidencia, Ios EE. UU. han seguido un acercamiento mucho más flexible y no han hecho movimientos directos para prevenir la proliferación horizontal80. Esta política alivió la tensión, pero no recuperó para los EE. UU. su antigua posición como principal socio nuclear de Brasil. El NPT de 1978 no permite al gobierno norteamericano dar licencia a expor. taciones nucleares a países no cubiertos por la totalidad de los mecanismos de control, y el Acta Internacional de Asistencia de Seguridad de 1977 prohíbe asistencia económica y militar a países que importan tecnologías de enriquecimiento y reprocesamiento, a no ser que hayan acordado colocar todas las instalaciones bajo auspicios multilaterales y sistemas de seguridad de IAEA. AI presidente se le permite solicitar la renuncia a esta acta, pero el presidente Reagan no lo ha solicitado en el caso brasileño. Esto dejó a la RFA en una posición dominante como proveedor nuclear.

\section{La República Federal de Alemania.}

La decisión de Alemania Federal de transferir un ciclo nuclear completo y ocho generadores a: Brasil estuvo basada principalmente en intereses comerciales $81 . \therefore$ Sin. embargo, los incentivos de recursos también han jugado un papet importante. Pobremente dotado con recursos naturales, la RFA encoṇtró en Brasil un socio que podía garantizar un aprovisionamiento de largo plazo de uranio natural ${ }^{82}$ :

De acuerdo con las condiciones del acuerdo, kwU ha estado colaborando desde 1975 con Nuclebrás en la construcción de reactores. Nuclebrás y la empresa alemana sTeAg han estado cooperando en el desarrollo del sistema de enriquecimiento por pitón a chorro Becker. La organización brasileña "Pronucleạ"" es responsable del entrenamiento técnico en Brasil, y la RFA és responsable de los técnicos involucrados en el programa. Nuclebrás tiene siete subsidiarias que

*Hubo sólo dos intervenciones directas. En 1981, la administración Reagan demandó - sin éxito - la sumisión de todo el IPEN al sistema de seguridad del IAEA ver Ferreira, p. 89; y anuló un acuerdo entre Princeton University y sus socios brasileños, que habria comprendido un proyecto de investigación conjuntá sobre tecnología de fusión nuclear; ver Myers, p. 900.

${ }^{3}$ Las motivaciones de Bonn se examinan en detalle en Helga Haftendorn, The Nuclear Triangle: Washingtgon, Bonn y Brasilia - National Nuclear Poliçes and International Proliferation (Washington D. C.: 1978), pp. 11-19 y Johnson. (1979), op. cit., :pp: 170-175.

"Mirow, op. cit., p. 132; Johnson (1979), op. cit., p. 173. 
operan en empresas conjuntas con una o más firmas de Alemania Federal ${ }^{83}$ A pesar de que se supone que Nuclebrás debía mantener los intereses de control, el control brasileño probó ser inexistente en el caso de Nuclebrás Engenharia (Nuclen). La prensa brasileña descubrió, aparentemente como resultado de una información filtrada por el gobierno norteamericano ${ }^{84}$, un tratado secreto entre KWU $\mathrm{Y}$ Nuclebrás estableciendo un derecho de veto para $\mathrm{kwU}$, a pesar de su participación minoritaria del 25 por cierito ${ }^{85}$.

El acuerdo trilateral de precauciones de IAEA, que fue firmado el 26 de febrero de 1976 también formó parte del paquete brasileñoalemán. Bajo este acuerdo, la RFA tiene que describir a la IAEA cualquier información técnica que provea a Brasil en las áreas principales del trato; desde ahí, cualquier planta construida usando esa información está sujeta a medidas de seguridad. La IAEA verifica que el material nuclear producido no sea usado con fines militares, para la producción de armas o de otros explosivos nucleares ${ }^{86}$.

EI vocero nuclear de Alemania Federal y la presidencia de Brasil trataron de desviar las preocupaciones de Estados Unidos al subrayar que estas medidas de seguridad eran más fuertes que aquellas requeridas por el $\mathrm{NPT}^{87}$. De conformidad con el acuierdo, las medidas de seguridad del raEa no sólo se extienden a los ftemes exportados y al material nuclear producido a través de su uso, sino también a cualquier instalación que Brasil pueda construir basada en la tecnología transferida y al material nuclear producido en ellas ${ }^{88}$.

La creciente crítica pública en torno àl acuerdo con la RFA, que ya había comenzado un corto tiempo después de la firma de éste, causó sólo reacciones moderadas de los alẹmanes. El kwU permaneció sorprendentemente callado durante los debates públicos. Después, de golpe sobre la pérdida de su trató con Irán, siguiendo a la

suclen (ingenierfa); Nuclam (exploración y prospección de uranio), Nuclep (componentes pesados); aparte de tres firmas alemanas; Voest-Alpine de Austria también participa, Intermational Herald Tribune (suplemento sobre energla nuclearr en América Latina), oct. de 1982, p. 6. Nuclei (cnriquecimiento y reprocesamiento de uranio), Nustep (investigación y desarrollo de técnologias de enriquecimiento de uranio), Nusteg (exploración de arena de monizita), y Nuclemon (planta de fabricación de combustible); para detalles ver Johnson (1979), op. cit, pp. 79-80.

${ }^{\text {s }}$ W. Grabendorff, "Bedingungsfaktoren und Strukturen der Nuklearpolitik Brasiliens", en Lothat Wilker (ed.), Nuklearpolitik im Zielkonflikt (Colonia: 1980), p. 64, NQ 70 .

\$TSTOE, $19 / 1 / 83$.

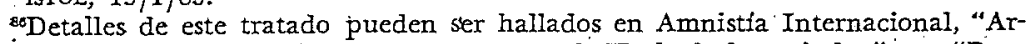
beitsgemeinschaft Katholischer Studenten und Hochschulgemeinden"; y "Bundesverband Bürgerinitiativen Umweltschutz", Das Deutsch-Brasilianische Atomgeschäft; 2da ed., (Colonia: 1977), pp. 29-30.

${ }^{87}$ Girotti, op. cit., 89-90; Spector (1984), op. cit., p. 24I; un ejemplo típico del escepticismo estadounidense puede encontrarse en.Filkelstein, op. cit., p. 284.

espector, (1984), op. cit., p. 241. 
revolución de 1978, la compañía estaba ansiosa de no contribuir de ninguna forma al deterioro del clima general en el cual se debía llevar a cabo el programa. Sin embargo, kwU insistió en 1983, en una nota a Nuclebrás, que la transferencia efectiỳa de tecnologia dependía de la construcción de más reactorès y no solamente de los dos primeros reactores en Angra, que habia sido firmemente contratado ${ }^{89}$. Esta intervención, interpretada por la prensa brasileña 6omo una amenaza, era apoyada por el presidente de Nuclebrás, Nogueira Batista, porque lo vela como un medio para presionar al gobiemo para que proporcionara los fondos necesarios destinados a, la la continuación del programa de Nuclebrás para la construcción de un reactor ${ }^{90}$.

El gobiemo de Alemania Federal adoptó un tono muy conciliador. El vocero de la embajada alemana aseguró a los brasileños en 1983 que "Alemania está más preocupada de.que Brasil se sobreponga a la crisis económica que del acuerdo nuclear"91. Este cauteloso acercamiento está motivado en general por los intereses comerciales y de inversiones de Alemania, que sobrepasan con creces el interés sectorial en la cooperación nuclear.

Después de los comentarios del presidente Sarney en diciembre de 1985 sobre la "necesidad de correcciones ëstratégicas" del progrạma nuclear, el gobierno de Alemania Federál adoptó una posición bastante pasiva, esperando las recomendaciones del comité reevalua. dor en el Ministerio de Minas y Energía. Jürgen Wap, Ministro de Gooperación Económica alẻmán, dijo en Brasilia que su góbierno esperaría las conclusiones del comité antes de comenzar las conversaciones conjuntas ${ }^{92}$. A pesar que esta afírmación deja abiêrta lá pregunta sobre si el gobierno de la RFA está listo para negociár todo el tratado o sólo el programa de su implementación, indíca claramente un alto grado de adaptabilidad a las nuevas circunstancias económicas y prioridades políticas. En las conversaciones venideras Bonn enfrentará un problema doble:

1) Si Bonn acepta proveer menos de ocho reactores en el largo plazo, el argumento original de que sólo ocho reactores asegüurarian la rentabilidad de las instalaciones de enriquecimiento y reprocesámiento se volvería obsoleto.

2) Si Bonn insiste, en cambio, en cumplir con el acuerdo original como propuso en 1979 el Ministro de Economía, Conde Otto Lambsdorff, Bonn se arriesgaría a perder su reputación en el Tercer Mundo de ser un socio económico flexible. Esta postura provo-

${ }^{30}$ ISTOE, $19 / 1 / 83$.

IIbid.

orbid.

Lotin American Regional Report, 9/8/85. 
carła retrasos aún más grandes en la implementación del programa, si es que no llevara a una resistencia política irrecuperable en contra del programa como un todo.

La imagen de la cooperáción nuclear de la RFA con Brasil quedaría incompleta sin mencionar la ayuda de Alemania Federal, además del tratado de 1975. En 198 I el Instituto Max-Planck ofreció, sin cargo alguno, una máquina pulsadora para ser usada por el equipo de fusión de la Universidad de São Paulo, que finalmente rechazó la oferta y continuó con sus propias investigaciones ${ }^{93}$. Se informa que en 1982 el IPEN recibió de Alemania Federal equipamento de protección de radiación, que po ibilitaba "que su planta de reprocesamiento extrajera plutonio en forma segura, al igual que asistencia tecnológica en la purificación de uranio ${ }^{94}$.

\section{Otros paises europeos}

Comparadas con las influencias determinantes de Ex.UU. y de la RFA durante las diversas fases del desarrollo nuclear de Brasil,' Francia y Holanda han ejercido sólo una influencia limitada.

La cooperación brasileña con Francia empezó en mayo de 1967 con un acuerdo de cooperación nuclear que incluía la investigación sobre reactores de torio $y$, aparentemente, el enriquecimiento de uranio por gas centrifugado ${ }^{95}$.

Dentro de los quince días siguientes a Ia firma del acuerdo con la RFA en 1975, el gobierno brasileño anunció la conclusión del acuerdo nuclear por el cual Francia aceptaba vender a Brasil un reactor experimental de generación rápida y cooperar en la exploración y desarrollo de sus depósitos de uranio ${ }^{96}$. El proyecto de explotación y molido de minerales en Poços de Caldas ya fue completado. La importación de un complejo de conversión francés para ser usado en la planta de enriquecimiento en Resende y el proyecto de reactor de generación rápida ha sido pospuesto indefinidamente $^{97}$. Ya que el último proyecto debía ser llevado a cabo por un consorcio franco-italiano, Brasil también terminó un acuerido con Roma para la construcción del reactor de generación rápida en el nuevo centro de investigaciones de Campo di Roma, en Itaguaiss.

Tanto los holandeses como los franceses han mostrado su preocu-

@ibid., $5 / 2 / 82$

Spector (1984), op. cit., p. 248; Myers, op. cit., p. 905; Latin American Regional Report, $5 / 2 / 82$.

${ }^{\infty}$ J. P. Rowles, "Nuclear Power and Nonproliferation: the View from Brazil", Vanderbilt Journal of Transnational Law, Vol. 14, No 4, otoño 1981, p. 746.

${ }^{\circ} \mathrm{E}$. W. Lefever, Nuclear Arms in the Third World - U.s. Policy Dilemma, (Washington D. C.: 1979), p. 107.

"Spector (1984), op. cit, 253; Spector (1985), op. cit., p. 205.

${ }^{a s}$ Latin American Regional Report, 5/2/82; Myers, op, cit., pP. 905-906. 
pación sobre la proliferación con respecto a Brasil. Hay dos casos en los cuales esto se demostró. EI primero estaba ligado al aprovisionamiento de combustible enriquecido a Brasil. Los alemanes esperaban proveer el combustible desde Urenco, pero esta opción hubiera sido factible sólo cuando la planta de enriquecimiento de Almelo (Holanda) fuera ampliada. Los socios holandeses se oponían a lá expansión con este propósito, a no ser que Brasil hubiera acordado almacenar el combustible usado no-separado (de este modo absteniéndose de reprocesarlo) bajo control multilateral y firmar el NPT.

Los brasileños rechazaron estas proposiciones, pero aún esperaban que la RFA cumpliera con todos los términos del tratado. De este modo, en abril de 1978, los alemanes aceleraron la construcción de su propia instalación de enriquecimiento en Gronau con el apoyo aparente de los socios británicos de Urenco. Este fue un factor importante que contribuyó a una nueva posición holandesa en junio de 1978, cuando los holandeses levantaron su veto a las exportaciones de combustible de Urenco a Brasil, a pesar de la imposibilidad. de esta última para estar de acuerdo con las demandas holandesas ${ }^{99}$. En el contrato que concluyó Brasil con Urenco un corto tiempo después, sin embargo, los holandeses tuvieron éxito en implementar e] control mulilateral sobre el combustible usado. El acuerdo provee que todo el p'utonio producido con uranio aportado por Urenco debe ser protegido; o por el establecimiento de medidas de seguridad universalmente aplicables por el IAEA para el almacenamiento de plutonio, o por un arreglo de almacenamiento ad hoc para tal plutonio bajo medidas de segur:dad del IAEA ${ }^{100}$.

Más aún, los brasileños declararon que buscarían llegar a un acuerdo con sus socios de Urenco sobre el posible reprocesamiento del combustible usado dos año; antes de que comenzaran los trabajos en la planta de reprocesamiento ${ }^{101}$.

La aceptación del control multilateral por parte de Brasil debe ser considerado como un logro importante para Holanda, cuya opinión pública es mucho más sensible a los temas sobre proliferación que aquella de los mayores proveedores europeos, en particular, de la RFA.

El segundo caso ocurrió en diciembre de 1983, cuando Brasil estaba negociando con Alsthom-Atlantique, el grupo francés de ingeniería controlado por el estado, para comprar los compresores nece-

"Para más detalles ver V. I. Johnson, "Brazil", en: J. E. Katz y O. S. Marwah (eds.), Nuclear Power in Developing Countries (Lexington; 1982), pp. 97-117.

${ }^{200}$ Reddick, op. cit., p. 132; Rowles, op. cit., p. $75 \mathrm{I}$.

${ }^{100} \mathrm{~J}$. A. Grandi, "Régime Militaire et Politique Extérieure du Brésil: L'accord de coopération nucléaire germano-brésilien de $1975^{\prime \prime}$, tesis doctoral sin publicar, Institut D'Etudes Politiques, Paris 1986, p. 421. 
Wolf Grabendorff / La politica nuclear y de no-proliferación de Brasil

Factores conjeturales

Internos democracia frágil

"absorción" inter-agencial

problemas financieros extre-

mos.

costo cambiante / relaciones

de beneficio

de diferentes fuentes de

energfa.
Factores estructurales

baja gradación de integración polktica.

problemas de gobernabilidad.

carencia de suficiente dominio tecnológico.

alternativa hidroeléctrica.
Externos

eficacia politica reducida de los Estados Unidos.

fortaleza económica de la

RFA.

competencia entre proveedores nucleares.

demanda por complejos nucleares de terceros países. alto grado de integración al mundo occidental.

dependencia de seguridad en caso de conflicto global.

dependencia tecnológica.

alto grado de integración al mercado mundial, particularmente a través de las multinacionales.

sarios para mejorar la eficacia de la planta de enriquecimiento por pitón a chorro en Resende. El Ministerio francés de Relaciones Exteriores se negó a dar las licencias de exportación necesarias, afirmando que se necesitaba tiempo para "reflexionar" sobre si se daría o no el permiso. Aparentemente estaban preocupados por los riesgos de proliferación al ayudar a Brasil a dominar el proceso de enriquecimiento. En especial las dudas sobre la justificación de la construcción de la planta de enriquecimiento, que habja sido diseñada para el aprovisionamiento de ocho reactores, pueden haber contribuido a la decisión francesa, ya que Brasil al mismo tiempo había decidido terminar sólo dos reactores que ya estaban en construcción en Angra, por lo menos durante un tiempo, y los contratos con Urenco cubrian la mayor parte de sus requerimientos de combustible. Los compresores fueron suministrados por empresas de Alemania Federal102.

\subsection{Toma de decisiones nucleares: resumen}

La complejidad del proceso de toma de decisiones nucleares en Brasil ha sido demostrada por la descripción anterior de su evolución y de las influencias externas. Es posible diferenciar entre factores estructurales y conjunturales por un lado, y entre factores ex-

200Para detalles comparar Spector (1984), op. cit., pp. 262-263. 
ternos e internos por otro. La tabla plantea los diversos grupos de factores que condicionan la toma de decis:ones en asuntos nucleares de Brasilios.

La importancia de los diversos factores varfa de acuerdo a los diferentes intereses de cada grupo. La transición desde un gobierno autoritario a una democracia, que aún continúa, ha llevado a un cambio de prioridades debido a los intereses de los nuevos grupos comprometidos en la toma de decisiones nucleares brasileñas, que difieren en gran medida de los intereses de los que trad:cionalmente toman las decisiones. La inclusión de más civiles que cumplen este papel bajo el gobierno de Figueiredo y de la comunidad científica bajo el gobierno de Sarney ha conducido a un énfasis en los problemas de Brasil para financiar el programa y en su alternativa hidroeléctrica.

La inestabilidad polftica, una gran dependencia del financiamiento externo y de la situación económica internacional, un alto grado de rechazo a cualquier tipo de interferencia política extranjera, y los problemas de ejercer un liderazgo político efectivo en una sociedad muy heterogénea, hacen que cualquier programa nuclear en Brasil sea candidato a grandes cambios. Como resultado, los países proveedores, en especial los socios nucleares de Brasil, tienen sólo una influencia mínima en la dirección de estos cambios. Brasil es probablemente uno de los mejores ejemplos de la exposición extrema a cambios de prioridades políticas o económicas de los programas nucleares en países del Tercer Mundo, que son considerablemente mayores que aquellos en los avanzados pafses induitrializados.

\section{Opciones de politicas nucleares}

Bajo un gobierno democrático, Brasil entra a su quinta fase de desarrollo nuclear una vez que la presente fase de revisión haya pasado. Dados los enormes problemas financieros y considerando el proceso de formación de fuerzas políticas que durará aún por algunos años más, es muy improbable que emerja pronto una polftica nuclear clara.

Las elecciones nucleares a futuro para Brasil se pueden agrupar en cuatro amplias opciones, que no son mutuamente excluyentes:

1) el uso de tecnología nuclear para generación de electricidad y para otros propósitos civiles, no explosivos;

2) el aprovisionamiento de otros estados con tecnología nuclear hecha en Brasil;

${ }^{100}$ Adaptado de Grabendorff (1980), op. cit., pp. 52-53. 
3) el desarrollo de explosiones nucleares pacíficas;

4) el despliegue de armas nucleares con las fuerzas armadas.

\subsection{Uso de energia nuclear}

Brasil consumirá electricidad generada por energia nuclear, aunque a niveles mucho más bajos de lo que se calculó en el tratado de junio de 1975. Este programa va a ser drásticamente reducido de escala, mientras que el programa paralelo va a ser mantenido con el fin ide obtener localmente capacidad de dominio de una amplia gama de tecnologias nucleares. El abandono del programa de energía nuclear sería un golpe significativo al prestigio político brasileño en América Latina y a través del mundo en desarrollo. Los beneficios políticos y tecnológicos y el valor de las diferentes fuentes de energía hacen que este compromiso valga la pena. Finalmente, las inversiones en tecnología nuclear de la actualidad van a poner a Brasil en una mejor posición para tomar ventaja de futuras tecnologías nucleares avanzadas y de adelantos que reduzcan costos. Consiguientemente, Brasil continuará buscando transferencias adicionales de tecnologías nucleares, pero en una forma mucho más selectiva que en el pasado.

\subsection{Brasil como proveedor nuclear}

Uno de los motivos para firmar el trato de junio de 1975 fue la creencia de que Brasil podría convertirse en un corto tiempo en un exportador importante de tecnología nuclear y uranio enriquecido. Pero, dadas las proyecciones drásticamente reducidas para la demanda de energía nuclear alrededor del mundo, el potencial comercial para las exportaciones nucleares brasileñas permanece incierto.

Brasil firmó en 1980 un acuerdo con Iraq, bajo el cual, en intercambio por petróleo, Brasil debía proveer uranio de baja intensidad y elementos combustibles terminados y además cooperar en la prospección de uranio y en los servicios de ingeniería para la construcción y seguridad de reactores. El acuerdo también incluía el entrenamiento de personal iraqui. Ante la insistencia brasileña y de acuerdo con las disposiciones del acuerdó brasileño-alemán-IAEA de 1976, el acuerdo brasileño-iraquí estaba sujeto a medidas de seguridad de IAEA. Cumpliendo con las restricciones en su acuerdo con la RFA, Brasil rehusó exportar materiales o tecnologías "sensibles"104.

Sin embargo, Israel estaba preocupado de que Iraq poseyera tecnología nuclear, y la implementación del acuerdo pudo haber jugado un rol en la decisión israelí de destruir el complejo nuclear de Bushir en $19 \dot{8} 1$.

${ }^{204}$ Texto del acuerdo en $O$ Estado de Săo Paulo, 25/1/80. 
Brasil adoptó otra importante decisión para la no-proliferación al requerir las medidas de seguridad del IAEA sobre las exportaciones nucleares de la República Popular de China, bajo un acuerdo de cooperación nuclear firmado por los dos estados el 11 de octubre de 1984. El acuerdo incluye la cooperación en la investigación y construcción de generadores de energía y reactores de prueba, la producción de elementos combustibles, seguridad nuclear, y la producción y aplicación de radio-isótopos ${ }^{105}$.

Hasta la fecha, Argentina ha sido el único comprador importante de tecnología nuclear brasileña. El acuerdo de cooperación nuclear de mayo de 1980 estipulaba que Nulcep debía construir el receptáculo del núcleo y los generadores de vapor para el generador de energía nuclear argentino de Atucha rr. La Comisión de Energía Nuclear Argentina debía a su vez suministrar a Brasil tuberías de zircalloy y tecnología para acopio de combu tible $e^{106}$. Debido a las dificultades financieras en ambos países, el acuerdo se ha implementado lentamente ${ }^{10 T}$.

En 1984; Brasil ganó una propuesta para construir en Somalía una planta de 60 millones de dólares de producción de concentrado de uranio ("yellowcake"), y recibieron ofertas de firmas turcas $y$ norteamericanas para la compra de "yellowcake". Sin embargo, Brasil no pudo terminar los últimos acuerdos porque carecía de los medios operativos para administrar el centro de producción de uranio en 'Poços Caldas 108 .

Brasil no ha abandonado su objetivo de convertirse en un proreedor nuclear. Las futuras exportaciones incluirán uranio, tanto tanto en su forma natural como enriquecido, y equipo pesado de Nulcep. Esto repre:enta uno de los pocos modos factibles para financiar por lo menos parte del desarrollo nuclear de Brasil. Pero es más que dudoso que Brasil realice alguna vez su sueño de convertirse en un exportador de reactores completos o de partes importantes de reactores para otros países en desarrollo.

Aparte del inexpugnable liderazgo argentino en esta área ${ }^{109}$, los brasileños enfrentarian una dura competencia de las firmas proveedoras del Norte. De este modo, la única opción factible para Brasll es establecerse como un proveedor internacional de componentes $y$ servicios selectos.

\footnotetext{
${ }^{106}$ Spector (1985), op. cit., p. 201; O Estado de Säo Paúlo, 5/12/84.

${ }^{100}$ International Herald Tribune, pp. 5-10; Filkelstein, op. cit., p. 302.

${ }^{107 M y e r s, ~ o p . ~ c i t ., ~ p . ~ 904 ; ~ L a t i n ~ A m e r i c a n ~ R e g i o n a l ~ R e p o r t, ~ B r a s i l, ~ 4 / 1 / 85 . ~}$

${ }^{105}$ Spector (1985), op. cit., pp. 201-2.

1.0Argentina proporcionó a Perú un reactor de investigación completo, Reddick, $o p$. cit., p. 127; ver también Courtney, op. cit., pp. 271-2.
} 


\subsection{Explosiones nucleares pacificas}

Los gobiernos brasileños han afirmado que las explosiones nucleares facíficas (PNE) podrían ser usadas para proyectos de remoción masiva de tierra en la cuenca del Amazonas, así como para soltar petróleö de reservas subterráneas. Dadas las dificultades ampliamente reconocidas envueltas en el uso de las PNEs, es muy improbable que Brasil intentara esto seriamente. Sin embargo, los esfuerzos diplomáticos del país al respecto han sido dignos de mención. Brasil ha manifestado que la implementación del artículo $\mathrm{v}$ del NPT (que establece un régimen internacional para los NNES) "sería equivalente a conferir el monopolio sobre esta tecnología a las potencias nucleares" 110 .

Brasil también ha apuntado a estudios del IAEA que revelan que Ios PNES podrian ser realmente significativos en la explotación de recursos naturales. Más aún, ha afirmado que la suposición básica del NPT de que no hay diferencia entre un arma nuclear y un aparato nuclear para explosiones pacificas, aún no ha sido establecida por consenso científico'11. En noviembre de 1975 el Ministro de Relacioneŝ́ Exteriores Azeredo da Silveira reiteró el "derecho" brasileño a llevar a cabo expløsiones nucleares pacificas con ocasión de la visita oficial a Brasilia del Ministro de Relaciones Exteriores de la RFA, Hans-Dietrich Genscher, sólo unos pocos meses después de la conclusión del acuerdo con la RFA ${ }^{112}$.

Desde que Brasil firmó el acuerdo trilateral de medidas de seguridad con el IAEA y la RFA, la mención en público de los PNES ha terminado. Pero aún si Brasil hubiera adherido estrictamente a ese acuerdo, la capacidad tecnológica para construir explosivos nucleares puede ser alcanzada dentro de pocos años en los inseguros complejos experimentales del programa paralelo. Sin embargo, si Brasil fuera a ejercitar la opción de los explo:ivos pacfficos, despertarfa sospechas regionales $\mathrm{e}$ internacionales de que lo hubiera hecho por razones militares. Dado este costo político, los dudosos beneficios económicos, y los enormes riesgos ambientales, no es probable que Brasil continúe con esta opción a futuro.

\subsection{La opción de las armas nucleares}

La cuarta opción política para Brasil es desarrollar e instalar armas nucleares. Dado que Brasil será técnicamente capaz de desarrollar explosivos nucleares dentro de la próxima década, una "pequeña

\footnotetext{
${ }^{10}$ Citado en A. Kapur, International Nuclear Proliferation: Multilateral Diplomacy and Regional Aspects (N. Y. 1979), p. 346.

imibid.

1'stankfurter Rundschau, 22/11/75.
} 
fuerza nuclear" puede ser lograda hacia fines de este siglo. Tal fuerza ha sido definida como capaz de lanzar un primer golpe crefble contra una potencia regional con una pequeña fuerza nuclear $y$ de infligir graves daños en la represalia ${ }^{118}$.

\section{Incentivos y contraincentivos a la proliferación}

\subsection{Dimensiones de seguridad}

Al examinar qué percepciones tienen las élites que toman las decisiones de las amenazas externas, se debe subrayar que Brasil, a diferencia de otros países examinados en este estudio y que están en los umbrales nucleares, no enfrenta ninguna amenaza externa seria. Brasil tiene una clara superioridad militar y no es blanco de amenazas convencionales realistas desde cualquier país o coalición de países de América Latina. Ejerce una considerable influencia económica y/o política sobre Chile, Uruguay, Bolivia y Paraguay. Considerando su bien desarrollada industria de armamentos, su independencia de la asistencia militar de los Estados Unidos, y los profundos cortes que afectan a las Fuerzas Armadas en Argentina bajo el gobierno de Alfonsin, cualquier incentivo para adquirir armas nucleares no se deriva de una amenaza convencional externa.

Queda la posibilidad, aunque hipotética, de la amenaza externa de un ataque nuclear. En Brasil, que no está protegido por el paraguas nuclear de los Estados Unidos, ha existido por mucho tiempo el temor de que Argentina pueda desarrollar su propia fuerza nuclear. Esta percepción, alimentada continuamente por el liderazgo argentino en tecnología nuclear, ha sido revivida especialmente por el éxito argentino en completar el ciclo del combustible nuclear. Como resultado, la construcción de armas nucleares se ha vuelto sólo una cuestión de voluntad política y de recursos para Argentina. Este logro, combinado con el anuncio argentino de la construcción de misiles supersónicos de mediano alçance "Mart́n Pescador ASIV-2"114, agravó "la paranoia de sectores que crean enemigos"115. El temor en los círculos gobernantes brasileños acerca de que los vecinos del sur posean armas nucleares llevó a un debate público sobre las intenciones nucleares argentinas, Io que ayudó a crear el clima de inseguridad necesario para justificar el propio programa experimental brasileño para adquirir esta capacidad.

Un ejemplo de la atmósfera creada por estas sospechas ha sido la rúbita crisis en las relaciones brasileño-argentinas en septiembre de 1985, causadas por supuestos comentarios del Ministro de la Arma-

usc Courtney, op. cit., 275.

IuPara detalles de este misil ver $O$ Estado de Säo Paulo, 3/1/85.

INT Wrobel, op. cit., p. 8. 
da, Pires Gonçalves de que Brasil no se quedaría detrás si Argentina construía la bomba ${ }^{116}$. La postura brasileña fue clarificada unos pocos días después por el General Bayma Denys, jefe del Gabinete Militar, quien reiteró que Brasil estaba atado por el TT y por lo tanto no tenía intencione; de producir una bomba atómica. Pero en la eventualidad de que otro país del continente lo hiciera, "el TT no sería ya válido y el tema sería vuelto a discutir"i17.

Dadas estas percepciones de una potencial amenaza nuclear desde Argentina, que se volvería particularmente fuerte en caso de un golpe militar en Argentina, surge la pregunta acerca de que si Brasil tiene una estrategia militar que incluye armas nucleares para propó itos de disuación y ataque mil:tar.

El concepto de los militares sobre seguridad nacional continúa teniendo principalmente dimensiones internas. Entonces, el eslabón entre la seguridad nacional y la seguridad global no es el $\mathrm{m}^{\circ} \mathrm{smo}$ para eI Brasil que para los EE. UU. y la URSS, donde una estrategia militar fundamentada en la disuación y en la destrucción mutuamente aregurada tiene efectos estabilizadores. Las armas nucleares poseen una muy baja utilidad para enfrentar las amenazas internas que están basadas en el desarrollo económico y social de Brasil. El presidente, General De Alençax Castelo Branco (1954-1967) hizo hincapié en esta baja utilidad al afirmar que el potencial nuclear de IOS EE.UU. Y la URSS ayudó a "rehabilitar" las armas convencionales, "el único tipo adecuado" para "guerras de liberación o guerras revolucionarias, insurrección, contrainsurrección y aún movimientos de guerrilla"1is.

Así, desde un punto de vista de seguridad, el único incentivo para adquirir la capacidad de desplegar una pequeña fuerza nuclear serja ser capaz de reaccionar rápido en el caso de que Argentina estuviera pensando hacerlo. Dada la situación, se puede concluir que Brasil no tiene una estrategia coherente que requiere una fuerza nuclear. Esto también está basado en el hecho de que las pre-condiciones técnicas para cambiar tal estrategia no existe. El desarroIlo de una "pequeña fuerza nuclear" requiere la adaptación o adquisición de sistemas de entrega, el establecimiento de bases y el desarrollo de sistemas de comando, control y comunicaciones.

En lo que respecta a sistemas de entrega, sin embargo, el reciente desarrollo de actividades espaciales en Brasil requiere atención. Varios miles de técnicos trabajan en el Instituto de Actividades Ecpaciales $^{119}$ (IAE) del Centro Técn'co Aeroespacial (CTA), pertene-

${ }^{110}$ VEJA, $11 / 9 / 85$, pp. 65-6.

IrThid., p. 66 .

${ }^{118}$ Citado en Courney, op. cit., p. 270.

${ }^{119}$ South, feb. 1985, p. 80. Después d, 1980, cuatro mil personas; ex ministro N. Freire Lavanere-Wanderley, "El pensamiento estratégico brasileño. El poder 
ciente a la Fuerza Aérea, que está oficialmente volcado al desarrollo de transportes de satélites. Lo último que ha sido desarrollado es "SONDA IV", un cohete que es propulsado por combustible sólido $y$ es capaz de transportar hasta $500 \mathrm{~kg}$. a una altitud máxima de 730 kilómetros 120 . Hasta ahora, han despegado cohetes desde "Barreira do Inferno", cerca de Natal, en el estado de Rio Grande do Norte ${ }^{121}$. El objetivo del Gobierno es poner cuatro satélites brasileños en órbita entre 1989 y 1993. Con este propósito el gobierno empezó en agosto de 1985 la construcción de un centro espacial en $\mathrm{Al}$ cantará, cerca de la desembocadura del Amazonas ${ }^{122}$.

Aunque no hay dudas sobre la importancia del programa brasileño de satélites para uso civil, no existe ninguna duda que estor satélites pueden ser fácilmente transformados en misiles de mediano alcance, transportando cabezas nucleares en vez de satélite 123 . El Mayor General Piva, Director del crA. af"rmó en 1984 que "es obvio que si podemos colocar satélites en órbita con un cohete es relativamente fácil... transformar este cohete en un misil balístico. Pero Iograr esto está aún a mucha di“tancia"124.

El DFVLR alemán (Deutsche Forschungs und Versuchsanstalt für Luft und Raumfahrttechnik), una institución pública de investigaciones para tecno'ogía aérea $v$ espacial, coonera muy de cerca con $e^{\prime}$ CTA en la implementación del programa espacial bracileño y narticularmente en desarrollar cohetes de lanzamiento125. La uRss ha acucado, por tanto, a Ia RFA de promover actividades en Brasil aue saben que no pueden realizar en su propio territorio ${ }^{126}$. Pero el minictro de la RFA para Ciencia y Tecnología. Heinz Riesenhuber, derTaró en agosto de 1984 que la participación de Alemania Federal en el programa espacial brasileño no conduciría a este país al uso de tecnología alemana con propósito militar por parte de Brasili27.

Los temores de que Brasil esté llevando a cabo el programa espacial no sólo para propósitos civiles sino también con propósitos militares han revivido recientemente con ocasión del acuerdo de coo-

aéreo espacial", Estrategia, № 63, marzo-abril 1980, p. 110: alrededor de diez mil personas.

${ }^{130}$ Para más detalles ver South, Ibid; O Estado de São Paulo, 9/12/83; Frank. furter Algemeine Zeitung, 14/8/85.

${ }^{121}$ Der Spiegel, No 33, 1982, p. 101.

${ }^{105}$ Frankfurter Algemeine Zeitung, i4 /8/85.

IrJohnson (1979), op. cit., pp. 174-5; Girotti, op. cit., pp. 191-2; (ed.) Arbeitskreis Phisik und Rustung, Diebundesrepublik, als heimlicher Waffenexporteur (Berlín: 1983), pp. 10-11.

1a"Citado en The Latin American Times, 30/1/84, p. 86.

${ }^{105}$ Arbeitskreis Phisik und Rustung (muy útil); ver Johnson (1979), op. cit., pp. 174-5 y Der Spiegel, No 33, 1982, p. 101.

${ }^{128}$ Reddick, op. cit., p. 113, No 52.

ar Jornal do Brasil, $25 / 8 / 84$. 
peración con la República Popular de China, firmado en 1985. Bajo aquel acuerdo, Brasil transferirá tecnología de combustible sólido a la República Popular de China (RPG). A cambio, la RPC transferirá a Brasil tecnología avanzada sobre combustibles líquidos (capaces de empujar pesadas cargas pagadas de hasta 1.000 kgs. a distancias más grandes que aquellas posibles con cohetes de combustible sólido) y sistemas de guía de cohetes, que podrían ser usados con modificaciones menores para misiles ofensivos ${ }^{128}$.

De este modo, desde el momento en que Brasil empiece a poner satélites en órbita; podría poseer un sistema de entrega viable, si es que decide llevar a cabo las modificaciones necesarias a los misiles y a los sistemas de guías. Con un radio de acción de alrededor de $1.450 \mathrm{kms}$., SONDA IV representa un misil balístico de mediano alcance con un gran potencial destructor para Argentina, ya que las tres ciudades principales de Argentina están situadas dentro de este ran$\mathrm{go}^{129}$.

Paradojalmente, el mayor incentivo para adquirir armas nucleares es al mismo tiempo el mayor desincentivo para Brasil. E1 temor de provocar una carrera de armas nucleares en América del Sur representa la principal razón para no armar una fuerza nuclear. Argentina, con su significativo liderazgo en el desarrollo y uso de la tecnología nuclear, sería la más probable ganadora de tal carrera. Más aún, la carrera tendría un efecto desestabilizador en el ambiente de seguridad sudamericano, dado que la adquisición de armas nucleares podría ser usada como un instrumento para la diplomacia coercitiva y podría aún llevar a una confrontación convencional o nuc'ear. EI temor de provocar estos resultados es el motivo preponderante para que Brasil se "vuelva nuclear" y una razón importante para pasar su programa nuc!ear militar antes de adquirir armas nucleares.

\subsection{Consideraciones acerca de la politica exterior.}

La autoimagen brasileña sobre los asuntos mundiales está basada en la suposición de que por virtud de su tamaño, población, recursos y poder industrial debiera lograr la hegemonía regional y el status de potencia mundial dentro de este siglo 130 . Brasil se ve como una potencia comparable con Canadá e India, y por tanto verticalmente móvil desde un status de potencia intermedia a un status de potencia mundial.

10 Globo, $13 / 1 / 86$.

${ }^{100}$ Distanciás del territorio brasileño desde Buenos Aires: 300 kms.; Córdoba: 650 kms.; Rosario: 425 kms!

${ }^{120}$ Ver G. Couto e Silva, Geopolitica do Brasil. (José Olympio), (2a edición, R. de J.: 1967). 
Entre los diversos grupos ideológicos en Brasil no existen dudas sobre el objetivo de obtener para Brasil una posición importante e independiente en el concierto internacional. Los puntos de vista difieren sólo en cuanto a la ba:e doméstica para tal postura independiente y a las posibles alianzas externas. De este modo, la creencia en el destino de Brasil para convertirse en una potencia importante, y por consecuencia su nacionalismo, es una expresión de la cultura política de Brasil como un todo y deben persistir también bajo un gobierno democrático.

Cuatro percepciones principales han influenciado la política exteriór brasīeña desde mediados de los años 60131:

1. Gran espacio, la percepción de ser el país más grande en el hemisferio sur, con oportunidades de desarrollo muy prometedoras;

2. Grandeza brasileña, la idea de una nueva cultura multirracial y tropical que se sobrepone a las diferencias entre razas, Sur-

Norte, Este-Oeste; y que tiene como atributo una misión civilizadora para esta cultura de luso tropicalismo;

3. Milagro brasileño, el milagro económico en Brasil, que entre 1965 y 1974 produjo un crecimiento industrial promedio de 13 por ciento al año y una alza de los ingresos per capita de un 7 por ciento anual ${ }^{132}$;

4. Nacionalismo pragmático, la versión brasileña de nacionalismo, que excluye los enemigos tradicionales y amigos automáticos por igual y es guiado por. la pragmática persecución de los intereses brasileños.

La aplicación de estas percepciones en un nivel global llevaron a Brasil a abstenerse de tomar compromisos que lo ataran y que consideraran que coartaban el desarrollo tecnológico independiente (tales como el NPT en el campo nuclear), para buscar la aplicación y desarrollo de avanzadas tecnologias, destinadas a diversificar sus energias y provisiones de armas, y para armar su propia industria de armamentos. Los acuerdos nucleares de 1975 con la RFA y Francia simbolizaron la persecución de la "opción europea" en estos esfuerzos, y fueron repetidas en el campo de la distribución de armas, donde Europa es Iejos el mayor proveedor.

La cuestión del status también ha motivado a los que toman decisiones en Brasil para buscar opciones nucleares: las fuerzas nuclea-

\footnotetext{
191W. Grabendorff y Nitsch, Brasilien: Entwicklungsmodell und Aussepolitik (Münich: 1977), p. 162.

${ }_{132}$ Ver informe del Banco Mundial de 1981, p. 70; Girotti, op. cit., p. 59, habla de "tropicalismo industrial".
} 
Wolf Grabendorff / La politica nuclear y de no-proliferación de Brasil

res son percibidas como de un valor inestimable en términos del prestigio nacional. Pero las numerosas actividades diplomáticas de Brasil en los niveles regionales, interamericanos y globales 183 y su peso económico como la octava economía más grande del mundo han asegurado que la consecución de sus programas nucleares no haya sido vista como el único medio para adquirir nuevo status internacional.

Estos incentivos que derivan de los objetivos de la política exterior brasileña persistirán bajo un gobierno democrático. Parecen muy poco probables los cambios a estas alturas, dado el objetivo, ampliamente compartido, de hacer de Brasil una gran potencia, el papel prominente jugado por políticos del "ancien régimen" (como el Presidente Sarney, el Ministro de Minas y Energía, Aureliano Chave:, y el Jefe de Gabinete, Marco Maciel) y la influencia aún presente de los oficiales militares que han jugado durante largo tiempo un papel importante en la política nuclear brasileña. Sus percepciones sobre cómo incrementar el prestigio internacional de Brasil y de cómo garantizar su seguridad no cambiaron de la noche a la mañana con la transición a un gobierno democrático.

La "Revolución de 1964" fue dominada por el concepto de "Seguridad y Desarrollo", que también fue aplicado a la política nuclear brasileña. Los militares incluyeron en su percepción de seguridad no sólo las amenazas militares externas sino también el evitar la dependencia excesiva de otros Estados y las amenazas internas a través de la "subversión ideológica"134. Desarrollo significaba el desarrollo económico y social, el progreso militar y el desarrollo de una posición más fuerte para Brasil en el concierto internacional.

Las élites que toman decisiones continuarán articulando una posición anti-statu quo para Brasil con respecto a su posición en el escenario internacional. Las demandas para una total participación en la toma de decisiones internacionales, y la soberanía sobre sus propios recursos y sobre todos los otros aspectos de su desarrollo serán mantenidos y reforzados por el peso de la deuda, que demuestra tan dolorosamente Ia dependencia brasileña.

Sin embargo, las opiniones sobre la utilidad de continuar con la opción nuclear para conseguir estos objetivos ha comenzado a cambiar debido a las urgentes necesidades sociales y a las políticas de ajuste, que requieren de otras prioridades. Esto ha sido demostrado por Ios "nuevos resultados diplomáticos" de Brasil que se concentran en reducir su vulnerabilidad externa, particularmente en los campos financieros y comerciales, y no reside más en las aspiraciones de Brasil de convertirse en una gran potencia. En los años ve-

\footnotetext{
1æ: Para una enumeración de esas actividades, comparar Grabendorff (1977), op. cit., pp. I64-5.

${ }^{134}$ Grabendorff (1980), op. cit., p. 59.
} 
nideros, las políticas que intensifican la autonomía brasileña jugarán un papel más importante. El desarrollo a través de transferencias masivas de tecnología y a altos costos ya no es factible. De este modo, persistirá la corriente que se aleja de la implementación del programa nuclear de 1975 y que avanza hacia el programa paralelo.

Entre los incentivos proporcionados por la política exterior para que Brasil adquiera armas nucleares, la rivalidad de siglos con Argent na ha jugado un papel importante. Ambos países tradicionalmente han querido ser la potencia dominante en América del Sur. No fue hasta el auge de los 60 s y los 70 s que Brasil alcanzó una posición de superioridad económica y de influencia regional no desafiada por Argentina. La rivalidad continúa en el campo nuclear, considerado por ambas naciones como esencial para un mayor prestigio nacional e influencia internacional.

A pesar de las continuas suspicacias sobre las intenciones argentinas, sin embargo, ha habido un cambio notorio en las posturas de ambos países. La emergente cooperación nuclear entre ellos está abriendo nuevas perspectivas para la no-proliferación en América del Sur.

\subsection{Cooperación nuclear con Argentina.}

Hasta el arreglo definitivo de las disputas de la represa en el río Paraná a través del acuerdo de octubre de 1979 entre Paraguay, Argentina y Brasil, había habido cooperación nuclear entre ambos países sólo en el campo internacional. Ambos buscaron limitar las restricciones en sus desarrollos nucleares, como en el caso de las negociaciones de Tlatelolco, cuando colaboraron para hacer más xigidos los procedimientos de ratificación y para desarrollar una defensa común del derecho de ilevar a cabo PNEs. Una pauta similar de coordinación prevaleció en las conversaciones de desarme en Ginebra o dentro de la Junta de Gobernadores del IAEA ${ }^{135}$.

Las disputas de Brasil y EE. UU. con respecto al acuerdo con la RFA desencadenaron el apoyo de Argentina para la posición brasileña y sugerencias para cooperación bilateral. En mayo de 1980 un acuerdo formai de cooperación nuc?ear fue firmaclo por el Presidente, Generai Figueiredo y el Presidente, General Jorge Videla. Incluia un intercambio de técnicos, entrenamiento de personal e intercambio de información en la fabricación de componentes, protección física de material nuclear, exploración y producción de uranio, seguridad nuclear e investigacione; sobre diseño de reactores. Esto proporcionó acceso a Argentina al Centro de Información Computarizada de Brasil y anticipó el abastecimiento de zirconio

${ }^{135}$ Reddick, op. cit., p. 130. 
argentino a Brasil y el eventual abastecimiento de uranio enriquecido brasileño para algunos reactores de investigación argentinos. Se formalizó un ạcuerdo por el cual Nuclep construyó la nave central para el tercer reactor argentino (que es suministrado por la $\left.\mathrm{RFA}^{138}\right)$.

Dada Ia rivalidad tradicional por la supremacfa continental entre los dos estados y la importancia que los círculos de poder de ambos países le han atr:buido siempre a sus programas nucleares para Пlenar sus aspiraciones geopolíticas, este acuerdo representó un paso importante hacia relaciones más predecibles entre ellos. EI acuerdo, que también contiene provisiones para una cooperación posterior, particularmente en asuntos relacionados con energía, planteaba grandes expectativas ${ }^{137}$. Parecía como si la competencia bilateral y la seguridad mutua hubieran sido superadas en favor de la comunicación y la cooperación, ayudando a asegurar a ambas partes contra sospechas y sorpresas 138 .

Sin embargo, excepto por el abastecimiento de óxido de uranio y tubos de zirconio de Argentina y la nave central de Brasil, ambas partes fueron lentas para implementar el acuerdo. La invasión argentina de las Islas Malvinas en abril de 1982 revivió las sospechas brasileñas sobre las intenciones argentinas y desde entonces este país ha sido subsecuentemente reacio a participar en empresas de energía nuclear con Argentina ${ }^{139}$.

Este momentum perdido fue renovado a principios de 1985, cuando el nuevo gobierno democrático de Argentina propuso un acuerdo por el cual todas las instalaciones nucleares sin excepción se abrirían para visitas recíprocas ${ }^{140}$. Esto abrió el camino para un acuerdo entre los presidentes Sarney y Alfonsín, en noviembre de 1985, para establecer un comité de trabajo conjunto para discutir Ias maneras de incrementar la cooperación bilateral con el objetivo de "multiplicar los beneficios del uso pacífico de la energfa nuclear" y de manejar las "crecientes dificultades encontradas en el abastecimiento internacional de equipamiento y materiales nucleares"141. Esto incluía la posible creación de un sistema local de salvaguardas

1salbid., pp. 131-2.

${ }^{237}$ Comparar, por ejemplo, Reddick, op. cit, p. 132, y Finkenstein, op. cit., pp. 302-3.

${ }^{135} \mathrm{E} 1$ presidente Figueiredo dijo en la ceremonia de firma del acuerdo "nuestro acuerdo refuta para siempre la leyenda de que Argentina y Brasil están empeñados en una carrera de armas nucleares y abre esperanzas para pasos concretos en una amplia área de intereses comunes" (citado en Finkenstein, op. cit., p. 303.

${ }^{130}$ Myers, op. cit., p. 904.

${ }^{130}$ International Herald Tribune, 29/3/85, p. 4.

1:1" Declaración conjunta de Ios señores presidentes de la Argentina y del Brasil del 30 de noviembre de 1985 sobre cooperación nuclear", informaciones de la Argentina, embajada argentina en Bonn, 4/12/85. 
mutuas. El comité de trabajo conjunto, que funciona bajo la coordinación de las respectivas Cancillerías, está formado por representantes de la comisión nacional y las empresas nucleares.

Como cada país en su pasado ha considerado al otro como la principal amenaza, justificando así su interés en la opción de las armas nucleares, esta declaración representa un importante paso hacia una mayor estabilidad regional. La cooperación planeada está supuesta de extenderse a "todos los campos del uso pacífico de la energía nuclear." que podría proveer a ambos países, con la seguridad de que ninguno está desviando su investigación para propósito militar.

Estimulada por la voluntad política de los actuales gobiernos democráticos en Brasil y Argentina, esta vez la cooperación nuclear bien puede convertirse en una realidad. Ambos países comparten objetivos comunes en sus políticas nucleares. Con respecto a temas de no-proliferación, los dos páses sostienen los mismos puntos de vista. Ellos perciben que al continuar in programa nuclear van a intensificar su status político y a mejorar su desarrollo tecnológico y económico. Para ambos paŕses, la independencia de los proveedores, con respecto al abastecimiento de combustible y a la transferencia de tecnología, se ha convertido en un objetivo importante.

\subsection{Consideraciones económicas.}

La situación financiera actual de Brasil, que tiene la deuda externa más alta del mundo, ejerce una múltiple influencia en el programa nuclear brasileño. Pero en contraste con los recortes en el programa de energla nuclear, no hay recortes conocidos en el programa nuclear paralelo; más' áún, el gobierno está empezando la construcción de un centro de control espacial para su ambicio.o programa espacial. Esto lleva a dos conclusiones:

1. La dependencia en el financiamiento, inversión y oportunidades comerciales externas es vista tanto por los militares como por los nuevos gobernantes civiles como una amenaza a la seguridad brasileña. Para evitar convertirse nuevamente en dependientes de la tecnología avanzada - tal como la tecnología nuclear, ei procesamiento de datos, la tecnologia espacial; y complejos sistemas de armas- "Brasil está "llevanido a cabo proyectos relacionados, a pesar de los gastos en que incurre.

2. Si la relativa posición económica de Brasil se debilitara y su influencia regional y su status internacional disminuyeran, las armas nucleares podrían ser súbitamente miradas como el único medio para mantener un alto status internacional. 


\section{Politicas de No-proliferación}

La proliferación de armas nucleares en el Tercer Mundo es vista por Brasil como algo más o menos irreversible. Si persiste esta tendencia, los incentivos del statu; padrían llevar a las élites brasileñas a la conclusión de que sólo la adquisición de una fuerza nuclear podría garantizar la posición internacional del país. Es esencialmente el deseo de mantener esta opción abierta lo que ha llevado a Brasil a adoptar y mantener su ambigua posición con respecto al TT.

Por el momento, Brasil continúa estando interesado en la participación activa en la tecnología nuclear, lo que no lleva necesariamente a la posesión de armas nucleares pero sí a la capacidad de producirlas.

Las siguientes precondiciones deben ser alcanzadas para que Brasil apruebe un esquema de no-proliferación ${ }^{142}$ :

1) obligaciones equivalentes y balanceadas para los paises con armas nucleares (NWS) y para los países que no tienen armas nucleares (NNWS);

2) un estricto vínculo entre el tratamiento de la proliferación horizontal y vertical;

3) la necesidad de los NNws de recibir garantías de seguridad internacional de los Nws.

\section{I. El Tratado de Tlatelolco.}

El 3 de noviembre de 1962, sólo días después del fin de la crisis de los misiles de Cuba, Brasil propuso en la Nu la creación de una zona latinoamericana libre de armas nucleares; esta iniciativa llevó en febrero de 1967 al Tratado de Tlatelolco (TT). Pero la actitud positiva de Brasil hacia una zona libre de armas nucleares camb:ó drásticamente bajo el gobierno militar el cual, a pesar de sus reservas, firmó y ratificó finalmente el tratado. Esto se debió a la inclusión del Artículo 18, que mantiene el derecho de conducir explosiones nucleares pacíficas ${ }^{143}$, y por la cláusula de "entrada en vigor", que requiere la ratificación de todos los países elegibles (a no ser que sea eliminado) para que el tratado llegue a ser totalmente efectivo. (Art. 28). Como Brasil, al igual que Chile y Argentina, no había

142Pilar Armanet, "EI OPANAL como instrumento de concertación política en América Latina", trabajo presentado en el Seminario América Latina: hacia nuevas formas de vinculación internacional, Arica, Chile, 18 al 22 de julio de 1984, pág. 25.

${ }^{1+2}$ El artículo 18 se incluyó después de esfuerzos de negociación común con. Argentina. 
eliminado esta cláusula, y Cuba y Guyana no han firmado el TT $^{\text {144, }}$ el tratado no ha entrado en vigor para Brasil.

Estas maniobras diplomáticas han dañado la reputación de Brasil, pero han mantenido abiertas para el país todas las posibles opciones. Sin embargo, Brasil siempre ha subrayado que se siente comprometido por el TT $^{145}$ y en junio de 1985 propuso una iniciativa en el Organismo para la Proscripción de las Ârmas Nucleares en la América Latina (OPANAL), la agencia que administra el TT, para crear mecanismos de verificación para controlar si los países con armas nucleares respetan realmente la zona libre de armas nucleares en América Latina ${ }^{146}$.

\subsection{El Tratado de No-Proliferación}

Brasil se ha opuesto al NPT desde su creación. Como miembro del Consejo de Desarme de las Naciones Unidas, este país presionó para que un tratado tomara en cuenta la seguridad de los países no-nucleares, pidió que se juntaran las medidas de no-proliferación con las de control de armamentismo de parte de las potencias nucleares, objetó la prohibición que tenían los países sin armas nucleares de efectuar PNEs, propuso salvaguardas obligatorias para todas las partes, y criticó el tratado por tener en forma general preferencias y discriminaciones ${ }^{147}$. Hoy en día, los principales argumentos de Brasil para no firmar el NPT son:

1) su negativa de abandonar sus intereses de seguridad de largo plazo para la garantía de los países con armas nucleares;

2) la consolidación de la actual distribución del poder mundial, que pone en peligro la movilidad ascendente de Brasil;

3) la discriminación del tratado entre tecnología "sensitiva" y PNES.

No hay indicaciones de que esta desaprobación fundamental del NPT pueda cambiar con un gobierno democrático, dado que estas percepciones son compartidas por una amplia mayoría del electorado.

\subsection{Salvaguardas del IAEA.}

Con respecto al IAEA, Brasil sigue una política muy diferente. Re-

\footnotetext{
14"Estas son las más notables excepciones: para una visión más detallada, comparar Armanet, op. cit., pp. 19, 24--5, 29; el artículo 28 ha sido extendido a la verificación de todos los poderes coloniales (protocolo 1) y de los NWs, (pro. tocolo 2), bajo los respectivos protocolos.

${ }^{14 s}$ Reddick, op. cit., p. 126.

${ }^{140}$ Jornal do Brasil, 16/6/85, p. 29.

${ }^{3 \times}$ Courtney, op. cit., p. 254.
} 
clama -en nombre propio y de los otros países en desarrollo- un papel activo del IAEA en el desarrollo de las instalaciones nucleares civiles; contempla las tareas de salvaguarda de la agencia como la menos importante ${ }^{148}$. Se cree que el control disminuye el status internacional de Brasil, mientras que la cooperación nuclear se percibe como una posición fortalecedora. Esto ha sido demostrado ep una forma muy particular por la activa participación de Brasil en el grupo de trabajo de los miembros latinoamericanos dél IAEA.

$\therefore$ La posición principalmente positiva de Brasil hacia el IAEA ha sido demostrada-específicamente por el término del trilateral Acuerdo de Salvaguardas con el IAEA y la RFA en febrero de 1976. Esta sumisión "voluntaria" a las salvaguardas del IAEA fue percibida en un caso específico como un acto que no disminuyó el status internacional de Brasil, mientras que la firma del NPT sería vista como una "áceptación "reductora de status" en la jerarquía internacional::

\subsection{Grupo de los Proveedores Nucleares.}

Brasil es muy crítico del trabajo del Grupo de Proveedores Nucleares y a sus directivas para el control de las exportaciones nucleares a un país no-poseedor de armas nucleares (NNWS). Los intentos de armonizar políticas de exportación nuclear han reforzado los temores de Brasil de verse impedido de adquirir tecnologías avanzadas. Más aún, el esfuerzo coordinado del grupo de prohibir la exportación de instalaciones para el enriquecimiento y reprocesamiento tóca exactamente aquellas áreas sensitivas de la transferencia de tecnología nuclear que son de interés especial para Brasil en su interito de demostrar su status e independencia.

\section{Gonclusiones.}

La revisión de la política nuclear brasileña será concluida pronto. Brasil está entrando a su quinta fase de desarrollo nuclear, que estará caracterizada por la continuación del programa de energía nuclear de 1975 a una escala bastante reducida, y la continuación del programa "paralelo". Esto refleja el deseo de Brasil do obtener una capacidad localmente desarrollada para dominar una amplia gama de- tecnologias nucleares. Al mismo tiempo refleja las limitaciones financieras del país y la menor necesidad de incrementar el abastecimiento de electricidad.

La crítica pública de este programa combinado se verá probablemente incrementada, a pesar de los cortes ya efectuados en la

${ }^{1+5}$ Wayne A. Selcher, Brazil's Multilateral Relations between First and Third World (Boulder, Colorado: 1978), p. 93. 
implementación del programa nuclear de 1975. Esto se debe al creciente poder de la izquierda brasileña, que no estará ni dispuesta $\mathrm{ni}$ en condiciones de justificar estas inversiones relacionadas con el status en contraste con las crecientes demandas sociales de su electorado. Otro factor es la mayor preocupación por el medio ambiente, que agrega otra dimensión al debate brasileño sobre energía. Ambos aspectos podrían ejercer una mayor influencia, dado el proceso dinámico de participación creciente en la política brasileña ${ }^{149}$.

La fuerte posición que los militares aún mantienen se verá reflejada en la consecusión de la opción de las armas nucleares. La adquisición de la capacidad para producir este tipo de armas no está realménte separada del programa nuclear brasileño, dada la importancia del proyecto paralelo para propósitos tanto civiles como posiblemente militares. Pero la explosión real de un artefacto nuclear sólo podría ser justificada por Brasil por razones de status político. No hay ninguna amenaza a la seguridad que requiera la búsqueda de esta opción. Sólo la explosión de un artefacto nuclear por parte de Argentina le darfa a Brasil la justificación necesaria. Si esto ocurriera, Brasil no vacilaría en construir explosivos nucleares.

Brasil no firmará el NPT, a menos que el tratado sea sustancialmente modificado. Es más probable que haya un fortalecimiento de los regímenes regionales de no-proliferación, o dentro del marco de opANAL, o en acuerdos bilaterales con Argentina, que podría ser extendido más tarde a otros Estados latinoamericanos. Esta opción tendría la excelente ventaja de excluir a las potencias nucleares ya establecidas y a los estados proveedores, y por tanto, de volverse más aceptable.

Considerando la. voluntad de Brasil de convertirse en un pro veedor nuclear importante, su disposición a entrar en conflictos políticos con sus socios nucleares del norte probablemente aumentará.

El fuerte involucramiento de empresas europeas en el desarrollo nuclear de Brasil estuvo motivada originalmente por la perspectiva de las ganancias a largo plazo, durante los años del auge brasileño. Los gobiernos europeos, en especial la RFA, mantuvieron a proveedores privados no sólo por intereses comerciales sino también porque estaban convencidos de que el mejor método para reducir los riesgos de proliferación sería faciljtar transferencias masivas de tecnologias bajo salvaguardas de amplio alcance. Desde comienzos de los años 80, sin embargo, Brasil se ha refrenado de hacer inversiones importantes en el campo de la tecnologfa nuclear. Por lo tanto, no hay oportunidad para una cooperación provechosa en este

${ }^{1 * 0} \mathrm{En}$ el estado de Río de Janeiro, por ejemplo, las estaciones de poder nuclear dependen desde 1984 de la aprobación por el Parlamento del Estado y un plebiscito; ver Jornal do Brasil, 5/9/84. 
campo para inversores europeos en el futuro previsible. Sin embargo, los gobiernos europeos y las empresas privadas probablemente continuarán la cooperación nuclear con Brasil, protegiendo de este modo sus intereses a largo plazo.

Dado que la historia de la proliferación nuclear demuestra que la adquisición de una capacidad de producir armas nucleares es inevitable una vez que los países se hayan comprometido seriamente a investigar en lo nuclear, hay muchas razones para que los europeos continúen su cooperación nuclear con Brasil. Una política que niega acceso a las tecnologías nucleares conduce a programas nacionales aislados y sin salvaguardas. Los europeos están convencidos de que su política de combinar la transferencia de tecnologías sensibles con salvaguardas estrictas es la mejor opción.

Al proveer a Brasil con abastecimientos garantizados de equipamiento nuclear y material bajo salvaguardas, los europeos pueden ser capaces de mantener algo de influencia sobre el desarrollo nuclear brasileño. Tal política también ayuda a invalidar el argumento brasileño de que la negativa de los estados proveedores de proporcionar material nuclear sensitivo y la inestable provisión de material nuclear requiere instalaciones nucleares locales y sin salvaguardas. Más aún, los proveedores europeos pueden ofrecer a Brasil la mayoría de las tecnologías con una base financiera mucho más atractiva, comparada con las enormes inversiones necesarias para el desarrollo local, de una amplia gama de tecnologías nucleares contempladas en el programa paralelo.

Otro importante factor es la actitud europea frente al status brasileño como un país en el umbral del desarrollo. Un reconocimiento formal de su posición como una de las potencias líderes del Tercer Mundo y como la octava economía más grande del mundo occidental puede satisfacer las demandas brasileñas de un creciente status internacional. La frustración continua sobre el "congelamiento del poder mundial"150 puede llevar a la adquisición brasileña de armas nucleares como un modo de incrementar su prestigio internacional. De este modo, un reconocimiento de la importancia brasileña en un nivel global bien puede reducir este riesgo. Las opciones posibles incluyen un asiento permanente para Brasil en el Consejo de Seguridad de las Naciones Unidas, su entrada al oECD, su participación en las cumbres económicas de las potencias líderes del mundo occidental, o su admisión como miembros del "Grupo de los 10" en el FMI. Las consultas preliminares sobre el tema desde el punto de vista europeo hasta ahora se han encontrado con una tenaz resistencia, especialmente de los EE. UU., quien parece opuesto a admitir a cualquier país del Tercer Mundo en estas instituciones.

${ }^{150}$ Grabendorff (1977), op. cit., p. 170. 
El cambio a un gobierno democrático en Brasil no varió la convicción de que el dominio del ciclo nuclear y la abstención del régimen establecido de no-proliferación le eran indispensables para convertirse en una gran potencia. Dadas estas continuas aspiraciones a ser una gran potencia y en vista de la importancia del status nuclear en las relaciones mundiales de poder, parece poco probable que cualquier gobierno brasileño - sea militar o democrático-deje pasar la oportunidad de mejorar su papel internacional a través del dominio de las tecnologías nucleares, a no ser que pudiera obtener beneficios sustanciales a cambio de un compromiso más fuerte hacia el régimen de no-proliferación.

\section{PRINCIPALES INSTALACIONES NUCLEARES EN BRASIL}

1. REACTORES DE ENERGiA (operando o en construcción).

Angra I* (agua liviana/uranio enriquecido de baja ley, $626 \mathrm{Mwe}$ )

proveedor: Westinghouse (EE. UU.).

inicio: 1985.

combustible: carga inicial: EE. UU,; recargas actuales Urenco (RFA, BU, Palses Bajos); uranio enriquecido en Brasil después de 1989 (est.).

Angra II* (agua liviana/uranio enriquecido de baja ley, 1.245 wwe).

proveedor: Krafterwerk Union (RFA),

inicio: 1992 (est.).

combustible: carga inicial, Urenco, uranio enriquecido en Brasil.

Angra II" (del mismo tipo de Angra II (aún no se sabe si va a ser completado).

2. RECURSOS DE URANIO / SITIOS MINEROS / MOLINÓS DE URANIó.

reservas razonablemente aseguradas: $310.000 \mathrm{t}$.

sitios mineros

: Poços de Caldas (inicio: 1982), Itataia.

molinos en operación

Poços de Caldas (producción anual: 500 t.
de uranio concentrado) e Itataia (inicio de plan piloto: 1986).

3. PURIFICACIÓN de uRanto.

IPEN, Såo Paulo.

capacidad: más de 10 t. por afio.

proveedor: Brasil con algo de equipo de la RFA.

inicio: 1981 . 
Wolf Grabendorff / La polftica nuclear y de no-proliferación de Brasil

4. GonÝersión a uRAinto heXfluorado.

\section{Resende*}

capacidad: $\quad 500$ t. planificadas p. a. (eventualmente 2.000 t. p. a.).

proveedor: Pechiney Ugine Kuhlmann (Francia).

Inicio:

pospuesto indefinidamente.

IPEN, São Paùlo (estala piloto).

capacidad: 90 t.p.a.

proveedor: Brasil.

inicio: 1984 .

IPEN, São Paulo (escala de laboratorio).

capacidad: (50 kgs. UF6 producidos hacia 1982).

proveedor: principalmente Brasil.

inicio: 1981 ó 1982.

5. ENRTQUDCIMIENTO.

\section{Resende}

tipo: pitón a chorro.

capacidad: $\quad 64$ t. de uranio enriquecido de baja ley en 1989.

proveedor: ... Krafterwerk Union (RFA).

inicio:

primera cascada en 1987 (est.). . .

Belo Horizonte*

$\begin{array}{ll}\text { tipo: } & \text { pitón a chorro. } \\ \text { capacidad: } & \text { escala de laboratorio. } \\ \text { proveedor: } & \text { Krafterwerk Union (RFA). } \\ \text { inicio: } & \text {. } 1980 .\end{array}$

IPEN, São Paulo.

tipo: ultracentrifugado.

capacidad: escala de laboratorio hasta 1990, después hay planes piloproveedor: Brasil.

inicio: 1987 , a escala de Iaboratorio (est.); 1990 plan piloto (est).

6. Fabricación de elementos combustubles.

\section{Resende*}

capacidad: $\quad 100$ t, planeadas p. a. para Angra I, II y Ií.

proveedor: $\mathrm{RFA}$.

inicio: 1982.

IPEN, São Paulo (plan piloto). 


\section{RIPROCESAMIENTO}

.

Resendè

capacidad: escala piloto, $10 \mathrm{kgs.}$ de combustible usado, por dia.

proveedor:

Inicio:

RFA.

pospuesto indefinidamente.

IPEN, São Paulo.

capacidad: (P).

proveedor: $\quad \therefore$ Brasil, con algo de equipamiento de la RFA.

Inicio: $\quad 1981$ (?).

salvaguardas: sólo cuando se esté procesando combustible con salvaguardas.

8. PRINCLPales REActores de INVESTigación

Instituto de Energía Atómica (IEA), São Paulo

nombre: $\quad$ IEAR $-I^{*}$

tipo: piscina / uranio altamente enriquecido, $5 \mathrm{MW}$ (th).

proveedor: 'Babock and Wilcolx, Martins Engel Company (EE. UU.).

Inicio: .. 1957.

Comissäo Nacional de Energia Nuclear (aNEN), Instituto de Engenharia Nuclear (IEN), Rlo de Janeiro.

nombre: $\quad$ IENR-1*

tipo: Argonaut / uranio de enriquecimiento mediano, $10 \mathrm{kw}$ (th).

proveedor: - Brasil (basado en diseños del Argonne National Laboratory; EE. UU.).

Inicio: 1965.

Instituto de Pesquisas Radiactivas (LRR), Belo Horizonte.

nombre: . Trigo-Mk-I*

tipo: Trigo I / uranio de enriquecimiento mediano, $100 \mathrm{kw}$ (th).

proveedor: General Dynamics Corporation (Us).

Inicio: ... 1960.

( $=$ bajo salvaguardas; $t .=$ toneladas).

FUENTES: Johnson (1979), pp. 68-9; Spector (1985), pp. 205-6; Der Spiegel $\langle 7 / 15 /$ 1985), pp. 94-5; South (Sept. 1985), p. 21; Nuclear Proliferation:Factbook, preparado para los Subcomités de Control de Armas, Seguridad Internacional y Giencia y - el de Política Económica Internacional y Comercio del Comité de Asuntos Exteriores, Cámara de Representantes de EE.uU. y el Subcomité de Energla, Proliferación Nuclear y Proceso Federal del Comité de Asuntos Gubernamentales, Senado de los EE,UU. por la División de Políticas de Medio Ambiente y. Recursos Naturales del Servicio de Investigaciones del Congreso, Biblioteca del Congreso, 990 Congreso, 1ra. Sesión (Washington D. C., 1985), pp. 346-351; Naçrichten für den Aussenhandel (enero 11 de 1985 y mayo 15 de 1986). 